\title{
PRIMFUNKTIONEN UND MULTIPLIKATIVE AUTOMORPHE FUNKTIONEN AUF NICHTGESCHLOSSENEN RIEMANNSCHEN FLÄCHEN UND ZYLINDERGEBIETEN.
}

\author{
VoN
}

KARL STEIN

in MÜNSTER.

Unter einer Primfunktion auf einer algebraischen Riemannschen Fläche $\mathfrak{A}$ wird gewöhnlich eine analytische Funktion von zwei unabhängig voneinander auf $\mathfrak{A}$ laufenden Variablen $\mathfrak{P}(\zeta)$ und $\mathfrak{P}(z)$ verstanden, die genau auf der ,Dia gonalmannigfaltigkeit" $\mathfrak{B}(\zeta)=\mathfrak{B}(z)$ in erster Ordnung verschwindet. Die Bezeichnung geht auf Weierstrass zurück, der in seinen Vorlesungen über Abelsche Transzendenten Primfunktionen zur Herstellung von Primfaktorzerlegungen algebraischer Funktionen verwendet hat. ${ }^{1}$ Später hat F. Klein den verwandten wichtigen Begriff der (auf homogene Variable bezogenen) Primform gebildet. Auch F. Prym hat Primfunktionen besonderer Art eingeführt und benutzt. ${ }^{3}$ Angesichts der Bedeutung, die den Primfunktionen auf algebraischen Riemannschen Flächen zukommt, scheint es von Interesse zu sein, entsprechende Funktionen und Verallgemeinerungen auch im nichtalgebraischen Falle zu studieren. Das soll in der vorliegenden Arbeit in Angriff genommen werden.

Auf einer geschlossenen Riemannschen Fläche können Primfunktionen sicher nicht als überall endliche und zugleich eindentige Funktionen gewählt werden. Die Primfunktionen von Klein und Prym sind zwar überall endlich, aber in bezug auf beide Veränderliche unendlich vieldeutig. Die Weierstrassche Primfunktion weist Pole und isolierte wesentliche Singularitäten auf; sie bleibt

1 Weierstrass, Werke Bd. 4, S. 387 ff.

${ }^{2}$ F. Kuein, Zur Theorie der Abelschen Funktionen. Math. Annalen 6 (I889/9o): Gesammelte Abhandlungen Bd. 3, S. 388 ff. - Vgl. auch W. F. OsGood, Lehrbuch der Funktionentheorie II, 2 ; S. 402 ff.

${ }^{3}$ F. Prym und G. Rost, Theorie der Prymschen Funktionen erster Ordnung, Leipzig 19I I, II. Teil. 
in bezug auf eine der beiden Veränderlichen eindeutig, nicht jedoch in bezug auf die andere Veränderliche. ${ }^{1}$ Es fragt sich, ob es nicht wenigstens auf einer nichtgeschlossenen Riemannschen Fläche $\Re$ stets eine überall endliche, eindeutige Funktion mit den Eigenschaften einer Primfunktion gibt. Hierzu wäre im Zylindergebiet $\mathfrak{R} \times \mathfrak{R}$ eine überall endliche, eindeutige Funktion $\Omega(\zeta, z)$ zu konstruieren, für die das analytische Flächenstück $\mathfrak{B}(\zeta)=\mathfrak{B}(z)$ als einzige Nullstellenmannigfaltigkeit, und zwar von erster Ordnung, vorgeschrieben ist.

Nun ist die Frage nach der Existenz analytischer Funktionen mehrerer Veränderlichen zu vorgegebenen Nullstellenflächen - bekannt als zweites Cousinsches Problem - in der Literatur eingehend behandelt worden. ${ }^{2}$ Seit längerem ist bekannt, dass es keineswegs immer möglich ist, ein in einem Gebiet $\mathfrak{B}$ über dem $R_{2 n}$ vorgelegtes Cousinsches Problem 2. Art durch eine eindeutige Funktion zu lösen. Damit eine eindeutige Lösungsfunktion existiert, müssen das Gebiet $\mathfrak{B}$ und die in $\mathfrak{B}$ vorgegebene Menge $\mathfrak{M}$ von Nullstellenflächen besondere Bedingungen erfüllen. Eine notwendige und in manchen Fällen auch hinreichende Bedingung besteht, wie ich in einer früheren Arbeit ${ }^{3}$ gezeigt habe, darin, dass die Schnittzahlen von $\mathfrak{M}$ mit den zweidimensionalen Zykeln in $\mathfrak{B}-$ ich nenne sie die charakteristischen $S$ chnittzahlen von $\mathfrak{M}$ in bezug auf $\mathfrak{B}$ - sämtlich verschwinden. Zur Diagonalmannigfaltigkeit des Zylindergebietes $\Re \times \Re$ gehören nun nur dann lauter verschwindende charakteristische Schnittzahlen in bezug auf $\Re \times \Re$, wenn $\Re$ schlichtartig ist. Daher kann es i. a. auf einer nichtgeschlosssenen Riemannschen Fläche eine überall endliche, eindeutige Primfunktion nicht geben.

Es lässt sich jedoch zeigen, dass als Primfunktion stets eine mehrdeutige, überall endliche analytische Funktion gewählt werden kann, deren Mehrdeutigkeitsverhalten dem der Weierstrasschen Primfunktion genau entspricht. Hierzu studieren wir das zweite Cousinsche Problem in allgemeinen Zylindergebieten $3=\mathfrak{R} \times \mathfrak{R}^{*}$, wo die nichtgeschlossenen Riemannschen Flächen $\mathfrak{R}$ und $\mathfrak{R}^{*}$ nicht

1 Die Weierstrassche Primfunktion hängt auch von den Polstellen analytisch ab; sie wird daher üblicherweise als Funktion von drei Veränderlichen geschrieben. Wir denken uns die Polstellenveränderliche festgehalten.

${ }^{2}$ Die erste grundlegende Untersnchung zu diesem Problemkreis stammt von P. Cousin: Sur les fonctions de $n$ variables complexes, Acta mathematica 19 (1895), S. I-6I. Ihre Ergebnisse mit Beweisen sind ausführlich dargestellt bei W. F. Osgood, Lehrbuch der Funktionentheorie II, I, Leipzig und Berlin. - Wegen weiterer Literatur sei auf den Bericht von H. BEHNKE und K. STEIN verwiesen: Analytische Funktionen mehrerer Veränderlichen zu vorgegebenen Null- und Polstellenflächen, Jahresbericht der deutschen Math. Vereinigung 47 (1937), S. I77--192.

3 K. STEIN. Topologische Bedingungen für die Existenz analytischer Funktionen komplexer Veränderlichen zu vorgegebenen Nullstellenflächen, Math. Annalen I I7 (I941), S. 727-757. 
notwendig als identisch vorausgesetzt sind. Wir beweisen, dass zu einer in 3 singularitätenfrei vorgegebenen Menge $\mathfrak{M}$ von Nullstellenflächen stets eine überall endliche, analytische Funktion gefunden werden kann, die in bezug anf die Veränderliche $\mathfrak{P}(z)$ eindeutig, in bezug auf die Veränderliche $\mathfrak{P}(\zeta)$ multiplikativ mehrdeutig ist, wobei die Multiplikatoren eindeutige nichtverschwindende Funktionen von $\mathfrak{B}(z)$ auf $\mathfrak{R}^{*}$ darstellen. Die charakteristischen Schnittzahlen von $\mathfrak{M}$ in bezug auf 8 hängen mit den Multiplikatoren eng zusammen und sind durch sie bestimmt. Wir nennen eine in 3 analytische Funktion $f(\zeta, z)$ mit solchem Mehrdeutigkeitsverhalten eine multiplikative automorphe Funktion (zu diesen gehören speziell auch die in 8 eindeutigen Funktionen). Insbesondere heisse $f(\zeta, z)$ eine Primfunktion, wenn sie genau auf einem in 3 irreduziblen Flächenstück in erster Ordnung verschwindet. Spezialfälle bilden die zur Riemannschen Fläche $\Re$ gehörenden Primfunktionen.

Sodann ergibt sich: Jede in 8 überall endliche multiplikative automorphe Funktion gestattet eine Darstellung als Produkt von Primfunktionen, und diese sind bis auf nichtrerschwindende multiplikative automorphe Funktionen als Faktoren eindeutig bestimmt. Dieser Sachverbalt entspricht dem Satz von der Primfaktorzerlegung der ganzen Funktionen. ${ }^{1}$

Wir zeigen weiter, dass in 3 stets überall endliche multiplikative automorphe Funktionen konstruiert werden können, zu deren Nullstellenflächen beliebig vorgegebene charakteristische Schnittzahlen gehören. Daher gibt es zu jedem Cousinschen Problem 2. Art in 3 eine eindeutige analytische Funktion, die wenigstens auf den vorgegebenen Nullstellenflächen in der vorgeschriebenen Ordnung verschwindet, eventuell jedoch noch weitere Nullstellen besitzt. Hieraus folgt, dass eine in 3 eindeutige meromorphe Funktion dort als Quotient eindeutiger, analytischer, überall endlicher Funktionen darstellbar ist, die jedoch nicht notwendig teilerfremd sind.

Es sei noch angemerkt, dass entsprechende Aussagen auch für Zylindergebiete mit mehr als zwei Projektionen gelten. Wir formulieren sie in einem ergänzenden Abschnitt.

\section{Inhaltsübersicht.}

г) Integrale I. Gattung und multiplikative automorphe Funktionen.

2) Multiplikative automorphe Funktionen zu vorgegebenen Nullstellenflächen.

3) Schnittzahlen und Multiplikatoren.

${ }^{1}$ Vgl. W. F. Osgood, Lehrbuch der Funktionentheorie II, I, 2. Aufl. S. 268 ff. 
4) Prim- und Elementarfunktionen Riemannscher Flächen.

5) Produktdarstellung multiplikativer automorpher Funktionen durch Primfunktionen.

6) Analytische Flächen zu vorgegebenen charakteristischen Schnittzahlen.

7) Multiplikative automorphe Funktionen in $2 n$-dimensionalen Zylindergebieten.

\section{I) Integrale 1. Gattung und multiplikative automorphe Funktionen.}

Wir stellen zunächst eine Reihe von Begriffen und Aussagen über Riemannsche Flächen und Funktionen auf Riemannschen Flächen zusammen, die im folgenden benötigt werden. Ein Teil dieser Aussagen wurde von H. Behnke und Verf. in einer kürzlich erschienenen Arbeit bewiesen. ${ }^{1}$

Unter einer Riemannschen Fläche wird stets eine über Punkten der komplexen Ebene ausgebreitete (konkrete) Riemannsche Fläche verstanden. Windungspunkte endlicher Ordnung sind als innere Punkte zugelassen. Sind Gs und $\mathfrak{S}_{1}$ Teilgebiete einer nichtgeschlossenen Riemannschen Fläche $\mathfrak{\Re}$ - in Zeichen $\mathscr{G}<\mathfrak{H}, \mathfrak{G}_{1}<\mathfrak{R}$ - so heisst $\mathfrak{G}$ ganz im Innern von $\mathfrak{G S}_{1}$ gelegen - in Zeichen

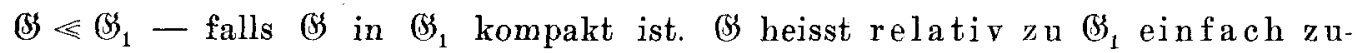
sammenhängend, wenn jedes endliche System geschlossener Kurven in (G), das innerhalb $\mathscr{S}_{1}$ berandet, schon in $(S)$ berandet. ${ }^{2}$ (S) heisst ferner ein Polygongebiet, wenn sein Rand aus endlich vielen punktfremden, doppelpunktfreien geschlossenen Kurven besteht, die ihrerseits aus endlich vielen Strecken und Halbgeraden zusammengesetzt sind, wenn ausserdem jeder Randpunkt Häufungspunkt äusserer Punkte ist. Ist eine Folge von Polygongebieten $\Re_{v}$ mit $\mathfrak{R}_{v} \ll \mathfrak{R}_{v+1} \ll \mathfrak{R}$ vorgelegt, derart dass es zu jedem Gebiet $\left(\mathfrak{S}^{*} \ll \mathfrak{M}^{*}\right.$ einen Index $\nu_{0}$ gibt, sodass für $\nu>\nu_{0} \mathscr{G}^{*}$ ganz im Innern von $\Re_{v}$ liegt, so heisst die Folge $\mathfrak{R}_{v}$ eine normale Ausschöpfungsfolge von $\mathfrak{R}$, wenn jedes $\mathfrak{R}_{v}$ relativ zu allen $\mathfrak{R}_{\mu}$ mit $\mu>\boldsymbol{\nu}$ und zu $\mathfrak{R}$ einfach zusammenhängend ist. Jede nichtgeschlossene Riemannsche Fläche $\mathfrak{R}$ besitzt normale Ausschöpfungsfolgen $\mathfrak{R}_{v}$, ebenso jedes Teilgebiet von $\Re$. Das Polygongebiet $\Re_{0}$ der normalen Ausschöpfungsfolge $\mathfrak{R}_{v}$ wird stets als schlechthin einfach zusammenhängend gewählt, es heisst der K e r $\mathbf{n}$ der Folge $\mathfrak{R}_{v}$. Sind ferner $\mathfrak{R}_{v}$ und $\mathfrak{R}_{v}^{*}$ normale Ausschöpfungsfolgen der nichtgeschlossenen Riemannschen Flächen $\mathfrak{R}$ bzw. $\mathfrak{R}^{*}$, so heisst entsprechend die Folge von Zylindergebieten $\beta_{v}=\mathfrak{R}_{v} \times \mathfrak{R}_{v}^{*}$ eine normale Ausschöpfungsfolge von $3=\mathfrak{R} \times \mathfrak{R}^{*}$ und $B_{0}=\mathfrak{R}_{0} \times \mathfrak{H}_{0}^{*}$ der Kern dieser Folge. Jeder nichtgeschlossenen

${ }^{1}$ H. Behnke und K. Stein, Entwicklung analytischer Funktionen auf Riemannschen Flächen, Math. Annalen I 20 (I948), S. 430-46I.

2 Es ist $\mathscr{G}<\mathscr{G}_{1}$ vorausgesetzt. 
Riemannschen Fläche $\mathfrak{A}$ und jedem Zylindergebiet 3 werde im folgenden jeweils eine normale Ausschöpfungsfolge fest zugeordnet.

Die eindimensionale Homologiegruppe $\mathfrak{S}^{1}$ von $\mathfrak{R}$, bezogen auf den Ring der ganzen Zahlen als Koeffizientenbereich ${ }^{1}$, besitzt eine höchstens abzählbare Basis. Falls $\mathfrak{R}$ nicht einfach zusammenhängend ist, kann und soll eine zugehörige Homologiebasis und die normale Ausschöpfungsfolge $\Re_{v}$ immer mit den folgenden Eigenschaften gewählt werden: Alle Basiszykel $\varepsilon_{\rho}, \varrho=1, \ldots$, sind einfach geschlossene, orientierte Kurven in $\Re$, die sämtlich einen festen Punkt $P_{0}$ des Kerns der Folge $\mathfrak{R}_{\nu}$ enthalten; ferner sollen jeweils $\mathfrak{S}_{1}, \ldots, \mathfrak{S}_{k_{v}}, \mathrm{o}<k_{1} \leqq k_{v} \leqq k_{v+1}$, eine eindimensionale Homologiebasis in $\mathfrak{R}_{v}, v \geqq \mathrm{I}$, bilden.

Auf $\Re$ existiert stets eine Elementarfunktion erster Ordnung. Hierunter wird eine im Zylindergebiet $B=\Re \times \Re$ eindeutige meromorphe Funktion ${ }^{2}$ $A(\zeta, z)$ der Veränderlichen $\mathfrak{P}(\zeta), \mathfrak{P}(z)$ verstanden mit der Eigenschaft, dass das Differential $A(\zeta, z) d \zeta$ für $\mathfrak{P}(\zeta) \neq \mathfrak{P}(z)$ endlich bleibt und für $\mathfrak{P}(\zeta)=\mathfrak{P}(z)$ einen einfachen Pol mit dem Residuum I aufweist. ${ }^{3}$ Ist $\mathbb{G} \& \Re$ ein Gebiet mit stïckweise glattem Rande und $f(\zeta)$ eine in (S) reguläre und auf dem Rande von (G) noch stetige Funktion, so ist $f$ in $(\mathcal{G}$ durch das Cauchysche Integral

darstellbar.

$$
f(z)=\frac{\mathrm{I}}{2 \pi i} \int_{\operatorname{Rd} \mathscr{S}} f(\zeta) \cdot A(\zeta, z) d \zeta
$$

Für Funktionen in Teilgebieten Riemannscher Flächen gilt der folgende Approximationssatz: Sind $\mathfrak{G S}_{1}$ und $\mathfrak{G}_{2}$ Teilgebiete der nichtgeschlossenen Riemannschen Fläche $\mathfrak{R}$ und gilt $\mathscr{G}_{1}<\mathscr{G}_{2}<\mathfrak{R}$, so sind die in $\mathscr{G}_{1}$ regulären eindeutigen Funktionen dann und nur dann stets durch in $\mathfrak{S H}_{2}$ reguläre eindeutige Funktionen gleichmässig im Innern von $\mathscr{G}_{1}$ approximierbar, wenn $\mathscr{G}_{1}$ relativ zu $\left(\mathfrak{G}_{2}\right.$ einfach zusammenhängend ist. Fin entsprechender Satz gilt für Funktionen mehrerer Veränderlichen in Zylindergebieten; die notwendige und hinreichende

\footnotetext{
1 Auch bei allen im folgenden benutzten Aussagen über Homologieeigenschaften von Riemannschen Flächen und Zylindergebieten ist der Ring der ganzen Zablen als Koeffizientenbereich zu Grunde gelegt. - - In bezug auf die topologische Terminologie folgen wir dem Lehrbuch der Topologie von H. SeIfert und W. ThReifall, Leipzig und Berlin 1934.

${ }^{2}$ Eine Funktion heisst in einem Punkte einer Riemannschen Fläche (oder eines Zylindergebietes) regulär bzw. meromorph, wenn sie es in hezug auf ortsuniformisierende Parameter ist.

s Elementarfunktionen sind im algebraischen Falle zuerst von WeikRstrass eingeführt worden. (Vorlesungen über Abelsche Transzendenten, Werke, Bd. 4). Zu diesem Begriff sei auchverwiesen auf R. KöNıg uud M. KRAFFT, Elliptische Funktionen, Berlin und Leipzig I928, sowie auf die weitere dort angegebene Literatur.
} 
Bedingung besteht darin, dass die Projektionen des kleineren Zylindergebietes relativ zu denen des grösseren einfach zusammenhängend sein müssen.

Auf einer nichtgeschlossenen Riemannschen Fläche $\Re$ lassen sich zu beliebig vorgegebenen Periodizitätsmoduln stets I ntegrale I. Gattung konstruieren. Damit ist folgendes gemeint: Werden den Elementen $c_{0}$ einer eindimensionalen Homologiebasis in $\Re$ beliebig komplexe Zahlen $\alpha_{\ell}$ zugeordnet, so gibt es eine auf $\mathfrak{R}$ uneingeschränkt regulär fortsetzbare Funktion $I(\zeta)$, die sich bei Fortsetzung längs $\mathfrak{C}_{\rho}$ oder einer zu $\mathfrak{C}_{Q}$ homologen Kurve additiv um die Konstante $\alpha_{\varphi}$ verändert. ${ }^{1}$ Ist speziell $\alpha_{\varrho_{0}}=\mathrm{I}, \alpha_{0}=$ o für $\varrho \neq \varrho_{0}$, so heisse ein zugehöriges Integral I. Gattung $I_{0_{0}}(\zeta)$ ein $\mathfrak{S}_{\rho_{0}}$ zugeordnetes Elementarintegral I. Gattung.

Über die Existenz von Integralen I. Gattung zu vorgegebenen Periodizitätsmoduln lässt sich noch eine weitergehende Aussage machen. Wir beweisen

Satz 1: $\mathfrak{R}$ und $\mathfrak{R}^{*}$ seien nichtgeschlossene Riemannsche Flächen. Den Elementen $\mathfrak{G}_{0}$ einer eindimensionalen Homologiebasis in $\mathfrak{A}$ seien Funktionen $f_{0}(z)$ zugeordnet, die auf $\mathfrak{\Re}^{*}$ eindeutig und regulär sind. Dann existiert im Zylindergebiet $8=\mathfrak{R} \times \mathfrak{R}^{*}=\mathfrak{R}_{\vdots} \times \mathfrak{R}_{z}^{*}$ eine uneingeschränkt regulär fortsetzbare Funktion $I(\zeta, z)$ mit folgenden Eigenschaften: Wird $\mathfrak{P}(z)$ festgehalten und durchläuft $\mathfrak{P}(\zeta)$ die Kurve $\mathfrak{E}_{\varrho}$, so verändert sich $I(\zeta, z)$ jeweils additiv um die Grösse $f_{\ell}(z)$; bei festgehaltenen $\mathfrak{P}(\zeta)$ und variablem $\mathfrak{P}(z)$ bleibt $I(\zeta, z)$ eindeutig.

$I(\zeta, z)$ heisse ein Integral 1 . Gattung auf $\mathfrak{R}$ mit dem Parameterbereich $\mathfrak{R}^{*}$, kurz: ein Integral I. Gattung in $8=\Re \times \Re^{*}$. Die $f_{\varrho}(z)$ heissen die Periodizitätsmoduln von $I(\zeta, z)$.

Beweis: Ist $\mathfrak{R}$ einfach zusammenhängend, so ist die Aussage des Satzes trivial; in diesem Falle ist schon $I(\zeta, z) \equiv 0$ eine gesuchte Funktion. Die Homologiegruppe $\mathfrak{S}^{1}$ enthalte also nicht nur das Nullelement. Es seien $I_{\varphi}(\zeta)$ den $\xi_{p}$ zugeordnete Elementarintegrale $I$. Gattung auf $\Re$. Besteht nun die eindimensionale Homologiebasis nur aus den endlich vielen Elementen $\mathfrak{c}_{1}, \ldots, \mathfrak{C}_{N}$, so hat

$$
I(\zeta, z)=\sum_{\eta=1}^{N} I_{0}(\zeta) \cdot f_{q}(z)
$$

${ }^{1} T(\zeta)$ ist durch seine Periodizitatsmoduln nur bis auf eine auf $\Re$ eindeutige Funktion bestimmt. - In verschiedenen neueren Untersuchungen ist der Begriff des Integrals 1. Gattung auf einer nichtgeschlossenen Riemannschen Fläche enger gefasst worden. Zn diesem Begriff vergleiche man: H. HorNich, Über transzendente Integrale erster Gattung, Monatshefte Math.-Physik 47 (1939), S. 380-387. - R. Nevandinna, Quadratisch integrierbare Differentiale auf einer Riemannschen Mannigfaltigkeit. Ann. Acad. Sci. Fennicae AI, Math.-Phys. r, (I94I), S. I-34. P. J. MyrkekG, Über transzendente hyperelliptische Integrale erster Gattung, Ann. Acad. Sci. Fennicae, AI, Nr. I4 (1943), S. I-32. Und: Über Integrale auf transzendenten symmetrischen Riemannschen Flächen, Aun. Acad. Sci. Fennicae 31 (1945). 
die geforderten Eigenschaften. Aber auch wenn unendlich viele $\mathbb{E}_{0}$ auftreten, kann ein $I(\zeta, z)$ als analog gebildete unendliche Summe gewonnen werden. Zur Sicherung der Konvergenz sind die $I_{0}(\zeta)$ zuvor geignet zu normieren: Die Kurve $\mathfrak{C}_{\rho}$ trete erstmalig als Basiskurve in $\Re_{v_{\rho}}$ auf, es sei also $0 \leqq k_{v_{Q}-1}<\varrho \leqq k_{v_{\rho}}$. Bei Fortsetzung innerhalb $\Re_{v^{-1}}$ bleibt $I_{Q}(\zeta)$ eindeutig; wir greifen einen $Z$ weig von $I_{\varphi}(\zeta)$ in $\Re_{v_{0}-1}$ fest heraus und bezeichnen ihn als Kernzweig $\hat{I}_{\varphi}(\zeta)$. Sei nun $\varepsilon_{q}$ eine Folge positiver Zahlen mit konvergenter Summe; ferner sei die reelle Zahl $c_{0}$ so gewählt, dass

$$
c_{\ell}>\operatorname{Max}_{\mathfrak{B}(z) \text { in } \mathbb{A}_{v_{\rho}}^{*}}\left|f_{\varrho}(z)\right|
$$

ist (es sei $\mathfrak{R}_{v}^{*}$ eine normale Ausschöpfungsfolge von $\mathfrak{R}^{*}$ ). Nach dem zitierten Approximationssatz gibt es eine in $\Re$ reguläre eindeutige Funktion $s_{q}(\zeta)$, sodass

Wir setzen

$$
\left|\hat{I}_{0}(\zeta)-s_{0}(\zeta)\right|<\frac{\varepsilon_{g}}{c_{\rho}} \text { in } \Re_{v_{e}-1}
$$

$$
J_{Q}(\zeta)=I_{0}(\zeta)-s_{0}(\zeta)
$$

für den Kernzweig $\hat{J}_{\varrho}(\zeta)$ in $\Re_{v_{e^{-1}}}$ gilt dann

Die unendliche Reihe

$$
\left|\hat{J}_{\rho}(\zeta)\right|<\frac{\varepsilon_{\rho}}{c_{\rho}} \text { in } \mathfrak{R}_{v_{\ell}-1}
$$

$$
I(\zeta, z)=\sum_{\ell=1}^{\infty} J_{\ell}(\zeta) \cdot f_{\ell}(z)
$$

konvergiert nun in jedem ganz im Innern von 8 gelegenen einfach zusammenhängenden Gebiet absolut und gleichmässig und stellt eine gesuchte Funktion in 3 dar; dabei sind jeweils solche $Z_{w e i g e}$ von $J_{\varrho}(\zeta)$ zu nehmen, die durch gleichzeitige Fortsetzung aus den Kernzweigen $\hat{J}_{\ell}(\zeta)$ hervorgehen. - Um dies zu zeigen, zerlegen wir:

$$
\sum_{\rho=1}^{\infty} J_{0}(\zeta) \cdot f_{\rho}(z)=\sum_{\rho \leq k_{v}} J_{\rho}(\zeta) \cdot f_{\rho}(z)+\sum_{\rho>k_{v}} J_{\rho}(\zeta) \cdot f_{0}(z)
$$

und weisen innerhalb $\beta_{v}=\Re_{v} \times \Re_{\nu}^{*}$ die Konvergenz der zweiten Summe rechts in (I) für die Kernzweige $\hat{J}_{\rho}(\zeta)$ nach. Es ist in $\beta_{v}$ für jedes $\varrho_{1} \geqq k_{v}$ 


$$
\left|\sum_{\rho>\rho_{1}} \hat{J}_{\rho}(\zeta) \cdot f_{\rho}(z)\right| \leqq \sum_{\rho>\rho_{1}}\left|\hat{J}_{\rho}(\zeta)\right| \cdot\left|f_{\rho}(z)\right| \leqq \sum_{\rho>\rho_{1}} \frac{\varepsilon_{\rho}}{c_{\rho}} \cdot c_{\rho}=\sum_{p>\eta_{1}} \varepsilon_{\rho} .
$$

Damit ist Satz I bewiesen.

Wir haben im folgenden häufig Funktionen von der Gestalt

$$
\tilde{M}(\zeta, z)=e^{I\{\xi, z)}
$$

zu betrachten; dabei sei $I(\zeta, z)$ ein Integral I. Gattung in $3=\mathfrak{R} \times \mathfrak{R}^{*}, \tilde{M}(\zeta, z)$ ist in 3 uneingeschränkt regulär fortsetzbar. Sein Mehrdeutigkeitsverhalten lässt sich wie folgt kennzeichnen:

I) Bei festem $\mathfrak{B}(\zeta)$ und variablem $\mathfrak{B}(z)$ bleibt $\tilde{M}(\zeta, z)$ eindeutig.

2) Wird $\mathfrak{P}(z)$ festgehalten und durchläuft $\mathfrak{P}(\zeta)$ eine orientierte geschlossene Kurve $\mathfrak{C}_{\wp}$ in $\mathfrak{R}$, so erhält $\tilde{M}(\zeta, z)$ einen nur von $\mathfrak{B}(z)$ und der Homologieklasse von $\mathfrak{S}_{o}$ in $\mathfrak{R}$ abhängenden Faktor $m_{q}(z)$; symbolisch

$$
\mathfrak{S}_{\rho}\{\tilde{M}(\zeta, z)\}=m_{\rho}(z) \cdot \tilde{M}(\zeta, z) ;
$$

dabei ist $m_{Q}(z)$ in $\mathfrak{R}^{*}$ regulär, eindeutig und ungleich Null.

Wir nennen allgemein eine in $8=\Re \times \Re^{*}$ uneingeschränkt regulär fortsetzbare Funktion $\boldsymbol{M}(\zeta, z)$ mit den Eigenschaften 1) und 2) eine auf $\Re$ reguläre multiplikative automorphe Funktion mit dem Parameterbereich $\mathfrak{R}^{*}$ - abgekürzt: eine in 3 reguläre m. a. Funktion -, auch dann, wenn $M(\zeta, z)$ sich nicht in der Gestalt (2) mit überall endlichem $I(\zeta, z)$ darstellen lässt. Eine in 8 reguläre m. a. Funktion heisse ferner eine Primfunktion in 3 , wenn sie auf genau einem irreduziblen analytischen Flächenstück in 3 in erster Ordnung verschwindet.

Besitzt die in 8 reguläre m. a. Funktion $M(\zeta, z)$ dort keine Nullstellen, so gestattet sie die Darstellung

$$
M(\zeta, z)=e^{I(\zeta, z)+J(z)}
$$

wo $I(\zeta, z)$ wiederum ein Integral 1 . Gattung in 8 (mit eindeutigen Periodizitätsmoduln) bedeutet und $J(z)$ ein Integral I. Gattung auf $\mathfrak{R}^{*}$, dessen Periodizitätsmoduln sämtlich ganzzahlige Vielfache von $2 \pi i$ sind. Dies ergibt sich aus

Satz 2: Die Multiplikatoren einer in $B=\Re \times \mathfrak{R}^{*}$ nichtverschwindenden, regulären multiplikativen automorphen Funktion $M(\zeta, z)$ besitzen eindeutige Logarithmen.

(Es ist jeweils selbstverständlich ein bestimmter Zweig des Logarithmus gemeint.) 
Beweis: Sei $\mathbb{C}$ eine orientierte geschlossene Kurve in $\Re$ und $\mathfrak{C}^{*}$ eine ebensolche Kurve in $\mathfrak{R}^{*}$; fèrner sei

$$
(\widetilde{M}\{(\zeta, z)\}=m(z) \cdot M(\zeta, z) .
$$

Wir bilden

$$
a(\zeta)=\int_{\mathfrak{E}^{*}} \frac{M_{z}^{\prime}(\zeta, z)}{M(\zeta, z)} d z=\int_{\mathfrak{E}^{*}} \frac{\partial \log M(\zeta, z)}{\partial z} d z
$$

Da $M(\zeta, z)$ eindeutig in bezug auf $\mathfrak{B}(z)$ ist, so ist für ein festes $\mathfrak{B}(\zeta): a(\zeta)=$ $=k \cdot 2 \pi i, k$ eine ganze Zahl. Andererseits hängt $a(\zeta)$ stetig (sogar analytisch) von $\mathfrak{B}(\zeta)$ ab; daher ist $a(\zeta)$ konstant. Es ist also

$$
(\widetilde{S}\{a(\zeta)\}=a(\zeta) \equiv k .2 \pi i \text {. }
$$

Nun ist nach (3):

$$
\left(5\left\{M_{z}^{\prime}(\zeta, z)\right\}==m^{\prime}(z) \cdot M(\zeta, z)+m(z) \cdot M_{z}^{\prime}(\overleftarrow{\zeta}, z)\right.
$$

also:

$$
\text { (7) } \begin{aligned}
\mathbb{S}\{a(\zeta)\} & =\int_{\mathbb{E}^{*}} \frac{\mathfrak{S}\left\{M_{z}^{\prime}(\zeta, z)\right\}}{\mathbb{E}\{M(\zeta, z)\}} d z \\
& =\int_{\mathfrak{E}^{*}} \frac{m^{\prime}(z) \cdot M(\zeta, z)+m(z) \cdot M_{z}^{\prime}(\zeta, z)}{m(z) \cdot M(\zeta, z)} d z \\
& =\int_{\mathbb{C}^{*}} \frac{m^{\prime}(z)}{m(z)} d z+\int_{\mathbb{C}^{*}} \frac{M_{z}^{\prime}(\zeta, z)}{M(\zeta, z)} d z \\
& =\int_{\mathbb{S}^{*}} d \log m(z)+a(\zeta) .
\end{aligned}
$$

Demnach muss, wegen (5),

$$
\int_{\mathfrak{S}^{*}} d \log m(z)=0
$$

sein, w. z. b. w.

\section{2) Multiplikative automorphe Funktionen zu rorgegebenen Nullstellen- flächen.}

Die Aufgabe, in einem Gebiet $(S)$ über dem $R_{2 n}$ zu vorgegebenen Nullstellenflächen eine in $(\mathscr{S}$ reguläre Funktion $z u$ konstruieren, kann nach Cousin wie folgt

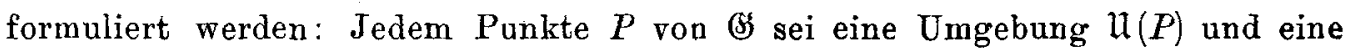


dort reguläre Funktion $f_{P}$ zugeordnet; im Durchschnitt $\mathfrak{U}(P) \cap \mathfrak{U}(Q)$ der Umgebungen zweier Punkte $P$ und $Q$ bleibe der Quotient $\frac{f_{P}}{f_{Q}}$ regulär und verschwinde dort nicht. Gesucht ist eine in $B$ reguläre Funktion $F$, sodass jeweils $\frac{F}{f_{p}}$ in $\mathfrak{U}(P)$ regulär und ungleich Null ist. Wir sagen, in Bf sei eine Cousinsche Verteilung 2. Art von regulären Ortsfunktionen vorgegeben; die gesuchte Funktion $F$ heisse eine zugehörige Lösungsfunktion. Reguläre Funktionen $f$ und $g$, für die in einem Gebiet $\mathfrak{U}$ gilt, dass der Quotient $\frac{f}{g}$ dort regulär bleibt und nicht verschwindet, nennen wir in $\mathbb{A}$ äquivalent in bezug auf Division, kurz $D$-äquivalent. Eine Lösungsfunktion zu einer Cousinschen Verteilung 2. Art muss also die Eigenschaft haben, mit allen Ortsfunktionen $D$-äquivalent zu sein.

Wir legen nun als Gebiet $\mathfrak{S O}_{\text {e }}$ ein Zylindergebiet $\$=\mathfrak{M} \times \mathfrak{R}^{*}$ zu Grunde, wo $\mathfrak{R}$ und $\mathfrak{R}^{*}$ wie bisher beliebige nichtgeschlossene Riemannsche Flächen über der $\zeta$ - bzw. $z$-Ebene bedeuten. Ist in 8 eine Cousinsche Verteilung 2. Art von regulären Ortsfunktionen vorgelegt, so existiert hierzu im allgemeinen, wie schon oben hervorgehoben, keine in 3 eindeutige Lösungsfunktion. Wir zeigen jedoch, dass als Lösungsfunktion stets eine reguläre multiplikative automorphe Funktion gewählt werden kann.

Sats 3: Im Zylindergebiet $8=\Re \times \Re^{*}$ sei eine Cousinsche Verteilung 2. Art von regulären Ortsfunktionen vorgegeben. Dann existiert eine in 8 reguläre multiplikative automorphe Funktion, die mit allen Ortsfunktionen äquivalent in bezug auf Division ist.

Beweis: Die Glieder der 3 normal ausschöpfenden Folge von Zylindergebieten seien mit $\beta_{v}=\Re_{v} \times \Re_{*}^{*}$ bezeichnet. Wir weisen zunächst in jedem (abgeschlossenen) Gebiet $\overline{\mathfrak{S}}_{v}=\overline{\mathfrak{A}}_{v} \times \overline{\mathfrak{R}}_{v}^{*}$ die Existenz einer regulären multiplikativen automorphen Funktion nach, die dort mit den vorgegebenen Ortsfunktionen $D$. äquivalent ist.

Hierzu werden $\overline{\mathfrak{M}}_{v}$ und $\overline{\mathfrak{R}}_{v}^{*}$ trianguliert, und zwar wird die Zerlegung von $\bar{\Re}_{*}$ and $\bar{\Re}_{v}^{*}$ in Dreiecke $\overline{\mathfrak{F}}_{i}$ und $\overline{\mathfrak{F}}_{j}^{*}$ so fein gewählt, dass jedes (abgeschlossene) Gebiet $\overrightarrow{\mathbb{E}}_{i j}=\overrightarrow{\mathfrak{E}}_{i} \times \overline{\mathfrak{E}}_{j}^{*}$ durch eine Umgebung $\mathfrak{U}_{i j}$ des durch die vorgelegte Verteilung von Ortsfunktionen bestimmten Umgebungssystems überdeckt wird; die zu $\mathfrak{H}_{i j}$ gehörige Ortsfunktion sei $f_{i j}(\zeta, z)$. Die Funktionen $f_{i j}(\zeta, z)$ sind nun in geeigneter Weise zu einer multiplikativen automorphen Funktion in $\overline{3}_{v}$ zu ,ver- 
heften". Der wesentliche Schritt dieses Heftungsprozesses besteht, in Analogie zum Vorgang von Cousin, in folgender Konstrultion:

Es seien $\overline{\mathfrak{\Re}}_{k}=\overline{\mathfrak{E}}_{1}+\cdots+\overline{\mathfrak{F}}_{k}$ und $\overline{\mathfrak{\Re}}_{l}^{*}=\overline{\mathfrak{E}}_{1}^{*}+\cdots+\overline{\mathfrak{E}}_{l}^{*}$ zwei zusammenhängende, als Punktmengen abgeschlossene Teilkomplexe vøn $\bar{\Re}_{v}$ bzw. $\overline{\mathfrak{R}}_{v}^{*}$; ferner sei $\overline{\mathfrak{F}}_{k+1}$ ein weiteres Dreieck der Triangulierung von $\overline{\mathfrak{R}}_{v}$, das mit $\overline{\mathfrak{K}}_{k}$ Randpunkte oder Randseiten, jedoch nicht alle drei Randseiten, gemeinsam hat. In $\overline{\mathfrak{\Re}}_{k} \times \bar{\Re}_{i}^{*}$ und $\overline{\mathbb{E}}_{k+1} \times \bar{\Omega}_{l}^{*}$ seien schon reguläre m. a. Funktionen $M_{1}(\zeta, z)$ bzw. $M_{2}(\zeta, z)$ als Lösungsfunktionen zur vorgegebenen Cousinschen Verteilung von Ortsfunktionen gefunden, und zwar seien die Multiplikatoren in $\overline{\mathscr{R}}_{l}^{*}$ regulär, eindeutig und ungleich Null. Gesucht ist eine entsprechende multiplikative automorphe Funktion in $\left(\overline{\mathfrak{R}}_{k}+\overline{\mathfrak{E}}_{k+1}\right) \times \overline{\mathfrak{K}}_{l}^{k}$.

Wir umgeben die $\overline{\mathfrak{\Re}}_{k}$ und $\overline{\mathfrak{E}}_{k+1}$ gemeinsamen Randstücke $R_{x}$ mit einfach zusammenhängenden, untereinander punktfremden Gebieten $\widetilde{\subseteq}_{k}$, die so klein gewählt sind, dass der Quotient

$$
Q(\zeta, z)=\frac{M_{1}(\zeta, z)}{M_{2}(\zeta, z)}
$$

jeweils in $\overline{\mathfrak{S}}_{x}=\overline{\mathfrak{S}}_{x} \times \overline{\mathfrak{K}}_{i}^{*}$ regulär und ungleich Null ist. In jedem $\overline{\mathfrak{5}}_{x}$ legen wir einen bestimmten Zweig $Q_{x}(\zeta, z)$ von $Q(\zeta, z)$ fest, dann ist $Q_{x}(\zeta, z)$ dort wegen des einfachen Zusammenhanges von $\widetilde{S}_{x}$ auch eindeutig. Es sei nun in $\overline{\mathfrak{S}_{x}}$

$$
G_{x}(\zeta, z)=\log Q_{x}(\zeta, z)
$$

wobei jeweils von einem bestimmten $Z_{w e i g}$ des Logarithmus ausgegangen sei. $G_{\varkappa}(\zeta, z)$ ist in bezug auf die Variable $\mathfrak{P}(z)$ in $\bar{\Re}_{l}^{*}$ ein Integral I. Gattung, seine Periodizitätsmoduln sind sämtlich ganzzahlige Vielfache von $2 \pi i$, (für die verschiedenen $G_{x}(\zeta, z)$ sind diese Periodizitätsmoduln möglicherweise verschieden). Wir wählen in $\bar{\Re}_{i}^{*}$ Integrale I. Gattung $a_{x}(z)$, derart dass $a_{x}(z)$ dort die gleichen Periodizitätsmoduln wie $G_{x}(\zeta, z)$ besitzt. Dann sei

$$
G_{x}^{*}(\zeta, z)=G_{x}(\zeta, z)-a_{x}(z)
$$

wobei rechts von bestimmten Zweigen ausgegangen werden soll. $G_{x}^{*}(\zeta, z)$ ist in $\overline{\mathfrak{S}}_{x}$ regulär und eindeutig.

$\overline{\mathfrak{S}}_{x}$ enthält ausser Punkten von $\overline{\mathfrak{K}}_{k}$ und $\overline{\mathfrak{E}}_{k+1}$ auch Punkte, die zugleich äussere Punkte von $\overline{\mathfrak{\Re}}_{k}$ und $\overline{\mathfrak{E}}_{k+1}$ sind. Wir können daher das $\overline{\mathfrak{\Re}}_{k}$ und $\overline{\mathfrak{E}}_{k+1}$ gemeinsame Randstïck $R_{x}$ zu einem gleichfalls noch in $\overline{\mathscr{S}}_{x}$ gelegenen doppelpunktfreien Kurvenstück $L_{\varkappa}$ ergänzen, dessen Endpunkte äussere Punkte in bezug auf $\bar{\Re}_{\varkappa}$ und $\mathbb{E}_{k+1}$ sind. Sodann bilden wix 


$$
g_{x}(\zeta, z)=\frac{\mathrm{I}}{2 \pi i} \int_{L_{x}} G_{x}^{*}(\xi, z) \cdot A(\xi, \zeta) d \xi,
$$

wo $A(\xi, \zeta)$ eine auf $\mathfrak{R}$ erklärte Elementarfunktion erster Ordnung bedeute. Die Funktion $g_{x}(\zeta, z)$ ist sowohl in $\mathscr{\Re}_{k} \times \overline{\mathfrak{\Omega}}_{i}^{*}$ wie in $\mathfrak{E}_{k+1} \times \bar{\Omega}_{i}^{*}$ definiert und dort jeweils eindentig und regulär. Bei Annäherung an $L_{x}$ innerhalb $\left(\mathfrak{\Omega}_{k}+\mathscr{E}_{k+1}\right) \times \overline{\mathfrak{M}}_{i}^{*}$ bleibt $g_{x}(\zeta, z)$ regulär, weist jedoch dort den additiven sprung $G_{x}^{*}(\zeta, z)$ auf; über die anderen $L_{\tau}, \tau \neq x$, ist $g_{x}(\zeta, z)$ dagegen ohne Sprung fortsetzbar. Demnach unterscheiden sich die Zweige von

$$
h_{x}(\zeta, z)=e^{\left.g_{x}(\xi) z\right)}
$$

am Kurvenstück $L_{x}$ jeweils um einen $Z$ weig von

$$
l_{x}(\zeta, z)=\frac{M_{1}(\zeta, z)}{M_{2}(\zeta, z)} \cdot e^{-a_{x}(z)}
$$

als Faktor; $L_{x}$ sei so orientiert, dass $h_{x}(\zeta, z)$ diesen Faktor erhält, wenn $\mathfrak{P}(\zeta) L_{x}$ von $\overline{\mathfrak{\Re}}_{k}$ nach $\overline{\mathfrak{E}}_{k+1}$ überschreitet. Es sei nun

$$
\begin{aligned}
& M_{12}(\zeta, z)=M_{1}(\zeta, z) \cdot \prod_{x} h_{x}(\zeta, z) \text { in } \Re_{k} \times{\overline{\aleph_{i}}}_{l}^{*} \\
& \tilde{M}_{12}(\zeta, z)=M_{2}(\zeta, z) \cdot \prod_{x} h_{x}(\zeta, z) \text { in } \mathfrak{F}_{k+1} \times \bar{\Re}_{l}^{*} .
\end{aligned}
$$

An den Kurvenstücken $L_{x}$ unterscheiden sich zwei bestimmte Zweige von $M_{12}(\zeta, z)$ und $\tilde{M}_{12}(\zeta, z)$ jeweils um den Faktor $e^{-a_{x}(\xi)}$. Daraus ergibt sich, dass $M_{12}(\zeta, z)$ von $\overline{\mathfrak{R}}_{k} \times \bar{\Re}_{i}^{*}$ in $\overline{\mathfrak{E}}_{k+1} \times \bar{\Re}_{l}^{*}$ hinein regulär fortsetzbar ist und dass das so fortgesetzte $M_{12}(\zeta, z)$ in $\left(\bar{\Re}_{k}+\overline{\mathscr{F}}_{k+1}\right) \times \bar{\Re}_{i}^{*}$ eine reguläre m. a. Funktion darstellt, die dort mit den Lokalfunktionen $D$-äquivalent ist und deren Multiplikatoren in $\bar{\Re}_{l}^{*}$ regulär, eindeutig und ungleich Null sind.

Wir denken uns nun die Dreiecke $\overline{\mathfrak{F}}_{k}$ und $\overline{\mathfrak{F}}_{l}^{*}$ der Triangulierungen von $\overline{\mathfrak{R}}_{v}$ und $\overline{\mathfrak{R}}_{v}^{*}$ so durchnumeriert, dass stets jeweils $\overline{\mathfrak{E}}_{k+1}\left(\right.$ bzw. $\left.\overline{\mathfrak{C}}_{l}^{*}\right)$ mit $\overline{\mathfrak{R}}_{k}=\overline{\mathfrak{E}}_{1}+\cdots+\overline{\mathfrak{E}}_{k}$ (bzw. mit $\overline{\mathfrak{R}}_{l}^{*}=\overline{\mathfrak{F}}_{1}^{*}+\cdots+\overline{\mathscr{F}}_{l}^{*}$ ) längs wenigstens einer Seite oder Ecke, niemals jedoch längs aller drei Seiten zusammenhängt. Dass dies für einen zusammenhängenden, endlichen Dreieckskomplex einer nichtgeschlossenen Riemannschen Fläche immer möglich ist, folgt in einfacher Weise durch vollständige Induktion nach der Anzahl der Dreiecke. Nach dem angegebenen Verfahren werden jetzt die Ortsfunktionen $f_{i j}(\zeta, z)$, die jeweils in $\overline{\mathfrak{E}}_{i} \times \overline{\mathfrak{E}}_{j}^{*}$ insbesondere eindeutig sind, in folgender Reihenfolge verheftet: Aus $f_{11}(\zeta, z)$ in $\overline{\mathscr{F}}_{1} \times \overline{\mathbb{E}}_{1}^{*}$ und $f_{12}(\zeta, z)$ in 
$\overline{\mathfrak{F}}_{1} \times \overline{\mathfrak{E}}_{2}^{*}$ wird (wobei die Variablen $\mathfrak{B}(\zeta)$ und $\mathfrak{B}(z)$ zunächst die Rollen vertauschen) eine Lösungsfunktion zur vorgegebenen Verteilung von Ortsfunktionen in $\vec{E}_{1} \times$ $\times\left(\overline{\mathfrak{E}}_{1}^{*}+\overline{\mathbb{E}}_{2}^{*}\right)=\overline{\mathbb{E}}_{1} \times \overline{\mathfrak{R}}_{2}^{*}$ konstruiert, hierauf je eine Lösungsfunktion in $\overrightarrow{\mathfrak{E}}_{1} \times{\overline{\mathfrak{R}_{3}}}_{3}, \ldots$, $\overline{\mathfrak{E}}_{1} \times \overline{\mathfrak{R}}_{l_{0}}^{*}=\overline{\mathfrak{E}}_{1} \times \overline{\mathfrak{R}}_{\nu}^{*}$, und ebenso in allen $\overline{\mathfrak{E}}_{k} \times \overline{\mathfrak{R}}_{\nu}^{*}$. Alle diese Lösungsfunktionen bleiben eindeutig, da die $\overline{\mathfrak{F}}_{k}$ einfach zusammenhängen. Aus den Lösungsfunktionen in $\overline{\mathfrak{F}}_{1} \times \overline{\mathfrak{R}}_{v}^{*}$ und $\overline{\mathbb{F}_{2}} \times \overline{\mathfrak{R}}_{v}^{*}$ wird sodann, wieder nach dem beschriebenen Verfahren, eine Lösungsfunktion in $\left(\overline{\mathbb{E}}_{1}+\overline{\mathbb{E}}_{2}\right) \times \bar{\Re}_{v}^{*}=\bar{\Re}_{2} \times \bar{\Re}_{v}^{*}$ konstruiert, hierauf eine in $\bar{\Re}_{3} \times \bar{\Re}_{v}^{*}$, u. s. f. So ergibt sich schliesslich eine in $\bar{B}_{v}=\bar{\Re}_{v} \times \bar{\Re}_{v}^{*}$ reguläre m. a. Funktion $M^{(v)}(\zeta, z)$, die dort mit allen Ortsfunktionen $D$ äquivalent ist.

Wir denken uns für jedes $v$ Funktionen $M^{(v)}(\zeta, z)$ wie angegeben gebildet. Damit aus ihnen eine Lösungsfunktion $M(\zeta, z)$ für das Gesamtgebiet 3 zusammengesetzt werden kann, sind sie zunächst geeignet zu normieren. Es seien (falls $\Re$ nicht einfach zusammenhängend ist) wie oben $\mathfrak{S}_{\varrho}, \varrho=\mathrm{I}, \ldots, k_{v}, k_{v} \leqq k_{v+1}$, in $\Re_{\nu}(\nu \geqq \mathrm{I})$ gelegene, zu einer eindimensionalen Homologiebasis in $\Re$ gehörende orientierte Kurven, die zugleich eine Homologiebasis in $\mathfrak{R}_{v}$ bilden. Ferner gelte:

$$
\mathfrak{S}_{g}\left\{\boldsymbol{M}^{(v)}(\zeta, z)\right\}=m_{v_{\rho}}(z) \cdot \boldsymbol{M}^{(\nu)}(\zeta, z) .
$$

Die $M^{(v)}(\zeta, z)$ werden nun so abgeändert, dass die Multiplikatoren nicht mehr von $v$ abhängen. (Ist $\Re$ einfach zusammenhängend, so ist eine Abänderung der $M^{(v)}(\zeta, z)$ nicht erforderlich).

Die multiplikative automorphe Funktion

$$
N^{(v)}(\zeta, z)=\frac{M^{(v+1)}(\zeta, z)}{M^{(v)}(\zeta, z)}
$$

ist in $\overline{3}$ regulär und ungleich Null; ihre Multiplikatoren

$$
n_{v \rho}(z)=\frac{m_{v+1, \varrho}(z)}{m_{v \rho}(z)}
$$

besitzen daher nach Satz 2 in $\overline{\mathfrak{R}}_{v}^{*}$ eindentige Logarithmen. Wir setzen in $\overline{\mathfrak{R}}_{v}^{*}$

$$
r_{v \rho}(z)=\log m_{v g}(z)
$$

wobei von einem bestimmten Zweige des Logarithmus ausgegangen sei; $r_{v}(z)$ ist ein in $\bar{\Re}_{v}^{*}$ definiertes Integral I. Gattung, dessen sämtliche Periodizitätsmoduln ganzzahlige Vielfache von $2 \pi i$ sind. In $\overline{\Re_{v}^{*}}$ ist jeder $\mathrm{Zweig}$ von $r_{v+1, \rho}(z)-$ $r_{\nu \rho}(z)$ also eindeutig, d. h. alle $r_{\mu \rho}(z)$ mit $\mu \geqq \nu$ besitzen in $\bar{\Re}_{v}^{*}$ die gleichen Periodizitätsmoduln. Sei nun $r_{\rho}(z)$ ein Integral I. Gattung auf $\mathfrak{\Re}^{*}$, das jeweils 
in $\Re_{v}^{*}$ die gleichen Periodizitätsmoduln wie $r_{\nu \rho}(z)$ besitzt; ferner sei $I_{\rho}(\zeta)$ ein $\mathbb{E}_{\rho} z u-$ geordnetes Elementarintegral I. Gattung auf $\mathfrak{R}$. Wir setzen in $\overline{\mathbb{B}}_{v}$ :

und

$$
H_{\nu \varrho}(\zeta, z)=e^{I_{Q}(g) \cdot\left[r_{\varrho}(z)-r_{v_{Q}}(z)\right]}
$$

$$
M^{*(v)}(\zeta, z)=M^{(v)}(\zeta, z) \cdot \prod_{\rho=1}^{k_{v}} H_{v}(\zeta, z),
$$

wobei rechts wiederum von bestimmten Zweigen der angegebenen Funktionen ausgegangen sei. Da

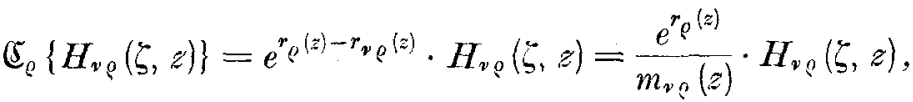

$$
\begin{aligned}
& \mathfrak{S}_{\sigma}\left\{H_{v_{\varrho}}(\zeta, z)\right\}=H_{v_{\varrho}}(\zeta, z) \text { für } \sigma \neq \varrho,
\end{aligned}
$$

so gilt wegen (8)

$$
\mathfrak{S}_{\tau}\left\{M^{*(v)}(\zeta, z)\right\}=e^{r_{\tau}^{(z)}} \cdot M^{*(v)}(\zeta, z), \quad \tau \leqq k_{v} .
$$

Die Multiplikatoren der $M^{*(v)}(\zeta, z)$ hängen daher von $v$ nicht mehr ab.

Aus den $M^{*(v)}(\zeta, z)$ wird nun mittels eines unendlichen Produktes eine gesuchte multiplikative automorphe Funktion im Gesamtgebiet $3=\Re \times \Re^{*}$ gebildet. Wir legen im einfach zusammenhängenden Kern $\overline{3}_{0}=\bar{\Re}_{0} \times \bar{\Re}_{0}^{*}$ der Gebietsfolge $\bar{\beta}$ je einen Zweig $\hat{M}^{*(v)}(\zeta, z)$ von $M^{*(v)}(\zeta, z)$ fest. Der Quotient

$$
\hat{N}^{*(v)}(\zeta, z)=\frac{\hat{M}^{*(v+1)}(\zeta, z)}{\hat{M}^{*(v)}(\zeta, z)}
$$

ist dann in $\bar{g}^{v}$ regulär, ungleich Null und bleibt dort eindeutig. Ferner ist

$$
K_{v}(\zeta, z)=\log \hat{N}^{*(v)}(\zeta, z)
$$

wo von einem bestimmten $Z$ weig des Logarithmus ausgegangen sei, in $\overline{3} v$ in bezug auf beide Variablen je ein Integral I. Gattung mit ganzzahligen Vielfachen von $2 \pi i$ als Periodizitätsmoduln. Es gibt je ein Integral I. Gattung $b_{v}(\zeta)$ in $\Re$ bzw. $b_{v}^{*}(z)$ in $\Re^{*}$ mit ganzzahligen Vielfachen von $2 \pi i$ als Periodizititsmoduln, derart dass

$$
K_{\nu}(\zeta, z)-\hat{b_{v}}(\zeta)-\hat{b_{v}^{*}}(z)
$$

in $\overline{3}_{v}$ eindeutig bleibt $\left(\hat{b}_{v}(\zeta)\right.$ bzw. $\hat{b}_{v}^{*}(z)$ seien in bestimmter Weise festgelegte "Kernzweige" von $b_{v}(\zeta)$ bzw. $\left.b_{v}^{*}(z)\right)$.

Sei nun eine Folge positiver Zahlen $\varepsilon_{v}$ mit konvergenter Summe vorgegeben. Wir wählen eine in $\bar{\beta}=\Re \times \Re^{*}$ reguläre Funktion $s_{v}(\zeta, z)$, derart dass in $\bar{B}_{v}$ : 


$$
\left|K_{v}(\zeta, z)-\hat{b}_{v}(\zeta)-\hat{b}_{v}^{*}(z)-s_{v}(\zeta, z)\right|<\varepsilon_{v}
$$

ist. Dann bilden wir

$$
M(\zeta, z)=M^{*(0)}(\zeta, z) \cdot \prod_{v=0}^{\infty}\left\{\frac{M^{*(v+1)}(\zeta, z)}{M^{*(v)}(\zeta, z)} \cdot e^{-b_{\nu}(\xi)-b_{\nu}^{*}(z)-s_{\nu}(\zeta, z)}\right\}
$$

wobei rechts solche Funktionen miteinander zu multiplizieren sind, die durch gleichzeitige Fortsetzung aus ihren Kernzweigen hervorgehen. Aus (9) folgt, dass das unendliche Produkt nach Abtrennung je endlich vieler Glieder in jedem $\overline{3}$ v absolut und gleichmässig konvergiert. $M(\zeta, z)$ ist also eine im Gesamtgebiet 8 definierte reguläre m. a. Funktion, die gemäss ihrer Konstruktion mit allen Ortsfunktionen $D$-äquivalent ist.

Damit ist der Satz bewiesen.

Es folgt insbesondere, dass zu einem in 3 vorgegebenen irreduziblen analytischen Flächenstück $\mathfrak{F}$ stets eine Primfunktion mit $\mathfrak{F}$ als Nullstellengesamtheit existiert. Denn zu $\mathfrak{F}$ kann stets eine Verteilung $D$-äquivalenter Ortsfunktionen angegeben werden, durch die $\mathfrak{F}$ als Nullstellenfläche 1. Ordnung vorge schrieben wird.

Ist wenigstens eine der Projektionen von $3=\Re \times \Re^{*}$ einfach zusammenhängend, so gibt es zu einer Cousinschen Verteilung $V$ von regulären Ortsfunktionen mit $D$-Äquivalenz sogar eine e indeutige reguläre Lösungsfunktion in 3 . Wird nämlich die Bezeichnung so gewählt, dass $\mathfrak{R}$ die einfach zusammenhängende Projektion von 8 ist, so ist die $V$ nach Satz 3 zugeordnete reguläre m. a. Funktion $M(\zeta, z)$ in 8 eindeutig.

Es sei noch angemerkt, dass in Satz 3 auch die bekannte Aussage über die Existenz einer auf einer nichtgeschlossenen Riemannschen Fläche $\Re$ regulären eindeutigen Funktion (einer Veränderlichen) mit vorgeschriebenen Nullstellen enthalten ist.

\section{3) Schnittzahlen und Multiplikatoren.}

In $3=\Re \times \Re^{*}$ sei wiederum eine Cousinsche Verteilung 2. Art $V$ von regulären Ortsfunktionen vorgegeben. Durch $V$ ist in 8 eine Menge von irreduziblen analytischen Flächenstücken $\mathfrak{F}_{n}$ bestimmt. Wir denken uns die $\mathfrak{F}_{n}$ in"natïrlicher" Weise orientiert; man erhält die natürliche Orientierung von $\mathfrak{F}_{n}$, indem man, von einer lokalen Parameterdarstellung ausgehend, die positive (d.h durch Linksdrehung bestimmte) Orientierung der Parameterebene auf $\mathfrak{F}_{n}$ über- 
trïgt. Jedem $\widetilde{F}_{n}$ ist ferner durch $V$ eine positiv-ganzzahlige Ordnung zugewiesen. Die Menge der so mit Orientierungen und Ordnungen versehenen Flächen heisse $\mathfrak{M}$.

Eine genau auf $\mathfrak{M}$ in den richtigen Ordnungen verschwindende reguläre m. a. Funktion $M(\zeta, z)$ ist selbstrerständlich nicht eindeutig bestimmt. Ist (mit den Bezeichnungen des vorigen Abschnittes)

$$
\widetilde{E}_{\rho}\{M(\zeta, z)\}=m_{\ell}(z) \cdot M(\zeta, z)
$$

so sind aber nach Satz 2 die Periodizitätsmoduln von

$$
r_{0}(z)=\frac{1}{2 \pi i} \log m_{p}(z)
$$

durch $\mathfrak{M}$ eindeutig festgelegt. Sie repräsentieren, wie sich im folgenden zeigen wird, topologische Invarianten ron $\mathfrak{M}$.

Wir bilden mit den Elementen $\mathfrak{S}_{\rho}, \mathfrak{C}_{s}^{*}$ der eindimensionalen Homologiebasen in $\Re$ und $\mathfrak{R}^{*}$ die Zykel $\mathfrak{C}_{g} \times \mathfrak{S}_{v}^{*}$ und bringen diese mit $\mathfrak{M}$ zum Schnitt. Legen wir als Orientierung von 3 die durch die positive Orientierung des $R_{4}$ bestimmte zu Grunde, so sind die Schnittzahlen $S\left(\mathfrak{M}, \mathfrak{E}_{Q} \times\left(\mathfrak{S}_{0}^{*}\right)\right.$ wohlbestimmt. Sie sind, wie wir beweisen wollen, mit den Periodizitätsmoduln der $r_{\varrho}(z)$ identisch. Es gilt

Satz 4: Sei $M(\zeta, z)$ ene regulare multiplikative automorphe Funktion in $B=$ $=\Re \times \Re^{*}$; es gelte

und

$$
\mathfrak{E}_{\mathrm{q}}\{M(\zeta, z)\}=m_{q}(z) \cdot M(\zeta, z)
$$

$$
a_{q \sigma}=\frac{1}{2 \pi i} \int_{E_{\sigma}^{*}} d \log m_{\ell}(z) .
$$

Dann ist

$$
a_{\rho \sigma}=S\left(\mathfrak{M}, \mathfrak{S}_{\rho} \times \mathfrak{C}_{\sigma}^{*}\right) .
$$

Beweis: Wir betten $\mathfrak{S}_{p}$ and $\mathfrak{S}_{\sigma}^{*}$ in Streifengebiete $\mathcal{G}_{p}$ bzw. $\mathcal{S}_{\sigma}^{*}$ ein, die ihrerseits ganz in $\mathfrak{R}$ bzw. $\mathfrak{R}^{*}$ liegen. Sodann bilden wir $\widetilde{\Xi}_{\text {" und }} \widetilde{\Xi}_{o}^{*}$ konform ab auf Kreisringe

bzw.

$$
\mathfrak{N}:\left\{1-\delta_{1}<|\zeta|<1+\delta_{s}\right\}
$$

$$
\Re^{*}:\left\{I-\delta_{2}<|z|<\mathrm{I}+\delta_{2}\right\} .
$$

$M(\zeta, z)$ geht hierbei in eine im schlichten Zylindergebiet $\tilde{3}=\Re \times \Re^{*}$ reguläre m. a. Funktion über, und die Grössen $a_{\rho \sigma}$ und $S\left(\mathfrak{M}_{i}, \mathfrak{C}_{\varphi} \times \mathbb{C}_{\sigma}^{*}\right)$ bleiben erbalten. 
Es genügt also, die Behauptung für m. a. Funktionen $M(\zeta, z)$ im Gebiete $\tilde{3}$ nachzuweisen. Als Kurven $\mathfrak{E}_{0}$, $\mathfrak{S}_{\sigma}^{*}$ seien hier die im Linkssinne durchlaufenen Peripherien der Einheitskreise gewählt; die übrigen Bezeichnungen seien beibehalten.

Zunächst wird $M(\zeta, z)$ geeignet normiert. Wir setzen

Dann ist

$$
M_{1}(\zeta, z)=M(\zeta, z) \cdot e^{\frac{-}{2 \pi i} \log \zeta \cdot \log \frac{\hat{z}^{a} \varrho 0}{m_{0}(\xi)}}
$$

$$
\mathfrak{\complement}_{g}\left\{M_{1}(\zeta, z)\right\}=z^{\alpha_{2 \sigma \sigma}} \cdot M_{1}(\zeta, z)
$$

Wir geben nunmehr eine spezielle m. a. Funktion mit dem Multiplikator $z^{-a_{\varrho \sigma}}$ an, deren Nullstellen übersehbar sind. Sei zunächst $a_{\sigma_{0}} \leqq$ o. Wir betrachten die analytische Fläche

$$
\mathfrak{F}: z=\zeta^{i}=e^{i \cdot \log }
$$

Ist $\mathfrak{F}$ in natürlicher Weise orientiert, so gilt, wie leicht ersichtlich,

$$
S\left(\mathfrak{F}, \mathfrak{S}_{?} \times \mathfrak{S}_{\sigma}^{*}\right)=+1
$$

$\mathrm{Zu}$ einer genau auf $\mathfrak{F}$ verschwindenden regulären m. a. Funktion gelangen wir wie folgt.

Wir setzen:

$$
H_{1}(\zeta, z)=\prod_{\nu=0}^{\infty}\left(\mathrm{I}-\frac{z}{e^{i \log \zeta+\nu \cdot 2 \pi}}\right), \quad H_{2}(\zeta, z)=\prod_{\mu=1}^{\infty}\left(\mathrm{I}-\frac{e^{i \log \zeta-\mu \cdot 2 \pi}}{z}\right),
$$

wo in allen Faktoren derselbe Zweig des Logarithmus genommen werden soll. Die unendlichen Produkte konvergieren für $\{0<|\zeta|<\infty ; \circ<|z|<\infty\}$ im Kleinen gleichmässig. Es sej dann

$$
H(\zeta, z)=e^{-\frac{\log ^{2} \zeta}{4 \pi}+\frac{\log \zeta}{1-i}} \cdot H_{1}(\zeta, z) \cdot H_{2}(\zeta, z) .
$$

$H(\zeta, z)$ verschwindet genau auf $\mathfrak{F}$ in erster Ordnung, und es gilt

$$
\aleph_{\varrho}\{H(\zeta, z)\}=z \cdot H(\zeta, z)
$$

In bezug auf die Variable $z$ ist $H(\zeta, z)$ eindentig. Bilden wir jetzt.

so gilt

$$
G(\zeta, z)=[H(\zeta, z)]^{-a_{\varrho \sigma}}
$$

$$
\mathscr{E}_{\varrho}\{G(\zeta, z)\}=z^{-a_{\rho} \sigma} \cdot G(\zeta, z) .
$$


Die Nullstellengesamtheit $\mathfrak{M}_{1}$ von $G(\zeta, z)$ ist das mit der Vielfachheit - $a_{0} \sigma$ versehene, natürlich orientierte Flächenstück $\mathfrak{F}$; es ist

$$
S\left(\mathfrak{M}_{1}, \mathfrak{C}_{\rho} \times \mathfrak{C}_{\sigma}^{*}\right)=-a_{\rho \sigma}
$$

Wir benötigen nun die Aussage, dass die Menge von Nullstellenflächen einer in einem Gebiet (S) regulären, eindeutigen Funktion mit jedem in G gelegenen zweidimensionalen Zykel die Schnittzahl Null besitzt. ${ }^{1}$ Wenden wir dies auf die in $\tilde{8}$ reguläre eindeutige Funktion

$$
\Phi(\zeta, z)=M_{1}(\zeta, z) \cdot G(\zeta, z)
$$

mit der Nullstellengesamtheit $\mathfrak{M}+\mathfrak{M}_{1}$ an, so ergibt sich

Andererseits ist

$$
S\left(\mathfrak{M}+\mathfrak{M}_{1}, \mathfrak{G}_{0} \times \mathfrak{E}_{\sigma}^{*}\right)=0 .
$$

$$
\begin{aligned}
S\left(\mathfrak{M}+\mathfrak{M}_{1}, \mathfrak{S}_{\rho} \times \mathfrak{S}_{\sigma}^{*}\right) & =S\left(\mathfrak{M}, \mathfrak{S}_{0} \times \mathfrak{c}_{\sigma}^{*}\right)+S\left(\mathfrak{M}_{1}, \mathfrak{S}_{0} \times \mathfrak{S}_{\sigma}^{*}\right) \\
& =S\left(\mathfrak{M}, \mathfrak{c}_{0} \times \mathfrak{c}_{\sigma}^{*}\right)-a_{\rho \sigma}=0 .
\end{aligned}
$$

Demnach ist

$$
S\left(\mathfrak{M )}, \mathfrak{S}_{\rho} \times \mathfrak{G}_{0}^{*}\right)==a_{\rho \circ}
$$

Ist $a_{\rho \sigma}>0$, so betrachten wir an Stelle von $\mathfrak{f}$ die Fläche

$$
\mathfrak{F}^{*}: z=\zeta^{-i}=e^{-i \cdot \log \xi}
$$

an Stelle ron $H(\zeta, z)$ die Funktion

$$
H^{*}(\zeta, z)=H\left(\frac{1}{\zeta}, z\right)
$$

und schliessen entsprechend.

Damit ist Satz 4 bewiesen.

Wir nennen die $a_{\rho \sigma}$ charakteristische Schnittzahlen von M; diese hängen also von der Wahl der Homologiebasen $\left\{\mathfrak{C}_{\varrho}\right\}$ und $\left\{\mathfrak{C}_{o}^{*}\right\}$ in $\mathfrak{A}$ bzw. $\mathfrak{\Re}^{*}$ ab.

Ist ein $a_{0_{1} \sigma_{2}} \neq 0$, so ist der zugeordnete Multiplikator $m_{\rho_{1}}(z)$ einer genau auf $\mathfrak{M}$ verschwindenden $m$. a. Funktion $M(\zeta, z)$ sicher nicht identisch $I ; m_{\rho_{1}}(z)$ hängt wirklich von $\mathfrak{P}(z)$ ab. Sind aber für ein $\varrho_{1}$ alle $a_{\rho_{1} \sigma}=0, \sigma=1,2, \ldots$, und ist gleichwohl $m_{\rho_{1}}(z) \neq \equiv \mathrm{I}$, so kann $M(\zeta, z)$ in nabeliegender Weise so abgeändert werden, dass bei Fortsetzung längs $\mathfrak{C}_{o_{1}}$ Eindeutigkeit herrscht. Allgemein gilt, dass $M(\zeta, z)$ ohne solche überflüssigen Mehrdeutigkeiten gewählt werden kann.

1 Vgl. die oben zitierte Arbeit des Verf., Math. Ann. 117, S. 734. 
Um das zu zeigen, betrachten wir die Menge derjenigen orientierten geschlossenen Kurven $\tilde{\mathbb{S}}$ in $\mathfrak{R}$, für welche

$$
S\left(\mathfrak{M}, \tilde{\mathfrak{S}} \times \mathfrak{G}_{\sigma}^{*}\right)=0
$$

für alle $\sigma$ ist. Diese $\tilde{\mathbb{C}}$ bestimmen eine Untergruppe der Homologiegruppe $\mathfrak{S}^{1}$ von $\mathfrak{R}$, die wir mit $\mathfrak{U}_{\mathfrak{d} \mathfrak{\mathfrak { R }}}^{1}$ bezeichnen. Dann gilt

Satz 5: Es sei $M(\zeta, z)$ eine in $3=\Re \times \Re^{*}$ reguläre multiplikative automorphe Funktion und $\mathfrak{M}$ die Menge ihrer mit Orientierung und Ordnungen versehenen Nullstellenflächen. Dann gibt es eine weitere in 3 reguläre m. a. Funktion $M_{1}(\zeta, z)$, die genau auf $\mathfrak{M}$ in den durch $M(\zeta, z)$ vorgeschriebenen Ordnungen verschwindet, mit folgender Eigenschaft: Es ist

$$
\tilde{\mathfrak{E}}\left\{M_{1}(\zeta, z)\right\}=M_{1}(\zeta, z)
$$

für jede in $\Re$ verlaufende geschlossene orientierte Kurve $\tilde{\mathbb{E}}$, deren Homologieklasse $z u \mathfrak{A}_{\mathfrak{y}}^{\prime}$ gehört.

Beweis: Besteht $\mathfrak{U}_{\mathfrak{d}}^{1}$ nur aus der Nullklasse, so ist nichts zu beweisen. In anderen Falle denken wir uns die Homologieklassen von $U_{\mathfrak{D} \imath}^{\mathfrak{1}}$ repräsentiert durch orientierte Kurven $\tilde{\mathfrak{E}}_{\tau}$. Zu jedem $\tilde{\mathfrak{E}}_{\tau}$ gehört ein Multiplikator $\tilde{m}_{\tau}(z)$ von $M(\zeta, z)$ :

$$
\tilde{\mathfrak{G}}_{\tau}\{M(\zeta, z)\}=\tilde{m}_{\tau}(z) \cdot M(\zeta, z) .
$$

Nach Satz 4 besitzen die $\tilde{m}_{\tau}(z)$ in $\mathfrak{R}^{*}$ eindeutige Logarithmen. Wir ordnen zu

$$
\tilde{\mathfrak{E}}_{\tau} \rightarrow g_{\tau}(z) \equiv \log \tilde{m}_{\tau}(z) \quad(\bmod 2 \pi i) .
$$

Diese Zuordnung bestimmt eine homomorphe Abbildung $A$ von $\mathfrak{U}_{\mathfrak{M}}^{1}$ in die additive Gruppe $\Gamma$ der in $\mathfrak{R}^{*}$ regulären eindeutigen Funktionen $\bmod 2 \pi i$ (wobei also Funktionen, die sich additiv um ein ganzzahliges Vielfaches von $2 \pi i$ unterscheiden, als gleich betrachtet werden sollen). Nach bekannten Schlussweisen der Gruppentheorie lässt sich dieser Homomorphismus zu einem Homomorphismus der gesamten Gruppe $\mathfrak{S}^{1}$ in $\Gamma$ erweitern. Jedem Element $\mathfrak{S}_{e}$ der eindimensionalen Homologiebasis in $\Re$ kann also eine in $\mathfrak{R}^{*}$ reguläre eindeutige Funktion $f_{\varphi}(z)$ zugeordnet werden, derart dass umgekehrt der durch die Zuordnung

$$
\mathfrak{\complement}_{\rho} \rightarrow f_{\varrho}(z) \quad(\bmod 2 \pi i)
$$

bestimmte Homomorphismus von $\mathfrak{H}^{1}$ in $\Gamma$ den Homomorphismus $A$ erzeugt. 
Nach Satz I existiert nun ein auf $\beta=\mathfrak{R} \times \mathfrak{R}^{*}$ definiertes Integral I. Gattung $I(\zeta, z)$ mit den Periodizitätsmoduln $f_{0}(z)$. Wir setzen

$$
N(\zeta, z)=e^{I(\xi, z)}
$$

$N(\zeta, z)$ ist eine in 3 reguläre nichtverschwindende m. a. Funktion, und es gilt

$$
\tilde{E}_{\tau}\{N(\zeta, z)\}=\tilde{m}_{\tau}(z) \cdot N(\zeta, z) \quad \text { für alle } \tau
$$

denn $I(\zeta, z)$ besitzt in bezug auf $\tilde{G}_{\tau}$ jeweils den Periodizitätsmodul $g_{\tau}(z)+k_{\tau} \cdot 2 x i$. Daher ist

$$
M_{1}(\zeta, z)=\frac{M(\zeta, z)}{N(\zeta, z)}
$$

eine in 3 reguläre multiplikative automorphe Funktion von der im Satz angegebenen Eigenschaft.

Folgerung: $Z u$ einer $i m$ Gebiet $3=\Re \times \Re^{*}$ gegebenen Cousinschen Verteilung 2. Art $V$ von regulären Ortsfunktionen existiont genau dann eine in B eindeutige reguläre Lösungsfunktion, wenn alle charakteristischen Schnittzahlen der durch $V$ bestimmten Menge analytischer Flächen verschwinden.

\section{4) Prim- und Elementarfunktionen Riemannseher Flächen.}

Wir wollen unsere Ergebnisse auf den Fall spezialisieren, dass die Projektionen $\mathfrak{M}$ und $\mathfrak{H}^{*}$ des $\mathrm{zu}$ Grunde liegenden Zylindergebietes 3 identisch sind. Es sei also

$$
3=\Re \times \Re
$$

wo $\Re$ wiederum eine nichtgeschlossene Riemannsche Fläche bedeute; wie bisher sei der laufende Punkt des ersten Faktors mit $\mathfrak{P}(\zeta)$, der des zweiten Faktors mit $\mathfrak{P}(z)$ bezeichnet. Die Diagonalmannigfaltigkeit

$$
\mathfrak{F}: \mathfrak{P}(\zeta)=\mathfrak{\$}(z)
$$

ist ein in 8 singularitätenfrei gelegenes irreduzibles analytisches Flächenstück. Eine Cousinsche Verteilung 2. Art von regulären Ortsfunktionen in 3, durch die $\widetilde{F}$ als Nullstellentläche . Ordnung vorgeschrieben wird, kann wie folgt gewählt werden: Falls der Punkt $P=\mathfrak{P}\left(\zeta_{0}\right) \times \mathfrak{P}\left(z_{0}\right)$ nicht auf $\mathfrak{F}$ liegt, setzen wir $f_{P}(\zeta, z) \equiv 1 ; \mathfrak{U}(P)$ wird so klein gewählt, dass es keinen Punkt von $\mathfrak{f}$ enthält. 
Liegt dagegen $P$ auf $\mathfrak{F}$, ist also insbesondere $\zeta_{0}=z_{0}$, und ist $\mathfrak{P}\left(\zeta_{0}\right)$ etwa Verzweigungspunkt $(v-\mathrm{I})$. Ordnung $(\nu \geqq \mathrm{I})$, so sei

$$
\begin{aligned}
& f_{P}(\zeta, z) \equiv\left(\zeta-\zeta_{0}\right)^{\frac{1}{v}}-\left(z-z_{0}\right)^{\frac{1}{v}}, \text { falls } \zeta_{0} \text { endlich, } \\
& f_{P}(\zeta, z) \equiv\left(\frac{\mathrm{I}}{\zeta}\right)^{\frac{1}{v}}-\left(\frac{\mathrm{I}}{z}\right)^{\frac{1}{v}}, \text { falls } \mathfrak{P}\left(\zeta_{0}\right) \text { unendlich ferner Punkt, }
\end{aligned}
$$

(wobei rechts die richtigen Zweige einander zuzuordnen sind); ferner sei $\mathfrak{U}(P)$ das direkte Produkt zweier $v$-fach gewundener, genügend kleiner Kreisumgebungen von $\mathfrak{P}\left(\zeta_{0}\right)=\mathfrak{P}\left(z_{0}\right)$ in $\mathfrak{R}$. Es existiert in 8 eine reguläre m. a. Funktion $\boldsymbol{\Omega}(\zeta, z)$, die, im Sinne von Satz 5 , ohne überflüssige Mehrdeutigkeiten gewählt sei. Wegen der Irreduzibilität von $\mathfrak{F}$ ist $\Omega(\zeta, z)$ eine Primfunktion; sie heisse eine Primfunktion der Riemannschen Fläche $\mathfrak{R}$.

Das Mehrdeutigkeitsverhalten von $\Omega(\zeta, z)$ wird nach Satz 4 und Satz 5 von den charakteristischen Schnittzahlen von $\mathfrak{F}$ in 3 bestimmt. Sind $\mathbb{S}$ und $\mathfrak{C}^{*}$ geschlossene orientierte Kurven auf $\mathfrak{R}$, so zeigt eine elementare Betrachtung, dass für die Schnittzahl $S\left(\mathfrak{F}, \mathbb{E} \times \mathbb{E}^{*}\right)$ die Beziehung

$$
S\left(\mathfrak{F}, \mathfrak{S} \times \mathfrak{S}^{*}\right)=S\left(\mathfrak{C}^{*}, \mathfrak{S}\right)
$$

erfüllt ist; hierbei werden zur Bildung von $S\left(\mathfrak{F}, \mathfrak{S} \times \mathfrak{C}^{*}\right) \mathfrak{S}$ und $\mathfrak{S}^{*}$ als Kurven auf zwei verschiedenen Exemplaren $\mathfrak{R}_{\xi}, \mathfrak{R}_{z}$ von $\Re$ betrachtet, während sie zur Bildung von $S\left(\mathcal{C}^{*}, \mathfrak{C}\right)$ als Kurven der gleichen, im üblichen Sinne positiv orientierten Riemannschen Fläche $\Re$ zum Schnitt gebracht werden. Ist insbesondere $\subseteq$ ein die Fläche $\Re$ zerlegender Rückkehrschnitt, so ist

$$
S\left(\mathfrak{C}^{*}, \mathfrak{C}\right)=0=S\left(\mathfrak{F}, \mathfrak{S} \times\left(\mathfrak{C}^{*}\right),\right.
$$

für beliebige $\mathfrak{C}^{*}$. Daher bleibt $\Omega(\zeta, z)$ eindeutig, wenn $\mathfrak{B}(\zeta)$ eine $\Re$ zerlegende Kurve durchläuft. Ist dagegen $(5$ ein nicht zerlegender Rückkehrschnitt, so gilt

$$
\mathbb{C}\{\Omega(\zeta, z)\}=\omega_{\mathfrak{S}}(z) \cdot \Omega(\zeta, z) .
$$

Dabei hat der Multiplikator $\omega_{\mathbb{G}}(z)$ folgende Eigenschaften: Die Funktion $\varphi_{\mathfrak{E}}(z)=\frac{\mathrm{I}}{2 \pi i} \log \omega_{\mathbb{E}}(z)$ (es sei von einem bestimmten $\mathrm{Z}_{\mathrm{weige}}$ des Logarithmus ausgegangen) stellt auf $\mathfrak{N}=\Re_{z}$ ein Integral I. Gattung dar; ihr Periodizitätsmodul ist $-\mathrm{I}$ in bezug auf den zu $\mathbb{E}$ konjugierten Rückkehrschnitt; Null jedoch 
in bezug auf jeden (5 nicht treffenden Rückkehrschnitt, ebenso in bezug auf $\mathbb{E}$ selbst, sowie in bezug auf jeden zerlegenden Räckkehrschnitt.

Aus der Primfunktion $\Omega(\zeta, z)$ lassen sich, ähnlich wie im algebraischen Falle $^{1}$, der Fläche $\mathfrak{R}$ zugeordnete Elementarintegrale und Elementarfunktionen gewinnen. So kann

$$
\Pi(\zeta, z)=\log \Omega(\zeta, z)
$$

als Elementarintegral 3. Gattung auf $\Re$ bezeichnet werden (wobei $\mathfrak{P}(\zeta)$ als Variable, $\mathfrak{P}(z)$ als Parameter aufzufassen ist). Die Periodizitätsmoduln von $\Pi(\zeta, z)$ in bezug auf nicht zerlegende Rückkehrschnitte stellen, wie eben dargelegt, Elementarintegrale I. Gattung auf $\mathfrak{R}$ dar. Schliesslich ergibt sich eine Elementarfunktion I. Ordnung auf $\Re$ als

$$
A(\zeta, z)=\frac{\partial}{\partial \zeta} \boldsymbol{\Pi}(\zeta, z)
$$

\section{5) Produktdarstellung multiplikativer automorpher Funktionen durch Primfunktionen.}

Im folgenden werde wieder ein allgemeines Zylindergebiet $3=\Re \times \Re^{*} z u$ Grunde gelegt, wo die nichtgeschlossenen Riemannschen Flächen $\Re$ und $\mathfrak{R}^{*}$ nicht als notwendig identisch vorausgesetzt sind. Wir wollen zeigen, dass eine reguläre m.a. Funktion nach ihren Nullstellenflächen in Primfaktoren zerlegbar ist, die durch Primfunktionen dargestellt werden. Es besteht also eine weitgehende Analogie zum Satze über die Primfaktorzerlegung der ganzen Funktionen.

Satz 6: Eine in Gebiet $3=\Re \times \Re^{*}$ reguläre multiplikative automorphe Funktion $M(\zeta, z)$ ist darstellbar als Produkt

$$
M(\zeta, z)=\prod_{n}\left\{M_{n}(\zeta, z)\right\}^{x_{n}}
$$

von Primfunktionen $M_{n}(\zeta, z)$ in 3. Das Produkt konvergiert, nach Abtreninung von je endlich vielen Gliedern, absolut und gleichmässig in jedem ganz im Innern von 3 gelegenen einfach zusammenhängenden Gebiet.

Das Produkt über die i. a. mehrdeutigen Funktionen $M_{n}(\zeta, z)$ ist dabei, ähnlich wie früher, so gemeint, dass solche $Z$ weige $\operatorname{der} M_{n}(\zeta, z)$ jeweils multipliziert werden sollen, die aus geeignet festgelegten Kernzweigen durch gleichzeitige Fortsetzung hervorgehen; unter dem Kernzweig von $M_{n}(\zeta, z)$ wird ein bestimmter

I Vgl. hierzu W. F. OsGooD, Lehrbuch der Funktionentheorie II, 2, S. 406. 
Zweig von $M_{n}(\zeta, z)$ im einfach zusammenhängenden Kern $3_{0}=\Re_{0} \times \Re_{0}^{*}$ der normalen Ausschöpfungsfolge $3_{v}=\mathfrak{R}_{v} \times \mathfrak{R}_{v}^{*}$ von 3 verstanden.

Zum Beweise des Satzes seien $\mathfrak{F}_{1}, \ldots, \mathfrak{F}_{n}, \ldots$ die irreduziblen Bestandteile der Menge $\mathfrak{M}$ von Nullstellenfächen von $M(\zeta, z) ; \lambda_{1}, \ldots, \lambda_{n}, \ldots$, seien die zugehörigen Ordnungen. Zu jedemı $\mathfrak{F}_{n}$ gehört eine Primfunktion $M_{n}^{*}(\zeta, z)$. Treten nun nur endlich viele $\mathfrak{F}_{n}$ auf, so ist

$$
M(\zeta, z)=N(\zeta, z) \cdot \prod_{n}\left\{M_{n}^{*}(\zeta, z)\right\}^{\lambda_{n}}
$$

eine Produktdarstellung von $M(\zeta, z)$; dabei ist $N(\zeta, z)$ eine geeignete nichtrerschwindende m. a. Funktion. Indem $N(\zeta, z)$ etwa mit $M_{1}^{*}(\zeta, z)$ zu einer Funktion $M_{1}(\zeta, z)$ zusammengefasst wird, ergibt sich eine gesuchte Darstellung. Es sei also weiterhin angenommen, dass $\mathfrak{M}$ aus unendlich vielen $\mathfrak{F}_{n}$ besteht; dann handelt es sich darum, die $\boldsymbol{M}_{n}^{*}(\zeta, z)$ zur Sicherung der Konvergenz des aus ihnen gebildeten unendlichen Produktes geeignet zu normieren.

Wie früher seien $\mathfrak{E}_{1}, \ldots, \mathfrak{C}_{k_{v}}$ in $\Re_{v}(v>0)$ gelegene, zu einer eindimensionalen Homologiebasis von $\mathfrak{R}$ gehörende orientierte geschlossene Kurven, die zugleich eine Homologiebasis von $\Re_{v}$ bilden; es gilt dann $k_{v} \leqq k_{v+1} \cdot{ }^{1}$ Entsprechend seien $\mathfrak{V}_{1}^{*}, \ldots, \mathfrak{S}_{l_{v}}^{*}$ in $\Re_{v}^{*}$ definiert. Jedem $\mathfrak{F}_{n}$ ist eindeutig ein $v(n)$ zugeordnet, derart dass $\mathfrak{F}_{n}$ nicht mehr in $\overline{3}_{v(n)}$, wohl aber in $\overline{3}_{v(n)+1}$ eindringt; aus $n \rightarrow \infty$ folgt dann $v(n) \rightarrow \infty$, da in jedes $\overline{\bar{\beta}} v$ nur endlich viele $\widetilde{F}_{n}$ eindringen können. Ohne Einschränkung der Allgemeinheit kann $\nu(n) \geqq I$ angenommen werden (hierzu brauchen höchstens endlich viele $\mathfrak{F}_{n}$ ausser Betracht bleiben). - Es gelte:

$$
\mathfrak{E}_{\rho}\left\{M_{n}^{*}(\zeta, z)\right\}=m_{n \rho}^{*}(z) \cdot M_{n}^{*}(\zeta, z) .
$$

Wir bilden nun in $\overline{3}_{v(n)}$ :

$$
G_{n}^{*}(\zeta, z)=\log M_{n}^{*}(\zeta, z)
$$

wo von bestimmten $Z$ weigen des Logarithmus und von $M_{n}^{*}(\zeta, z)$ ausgegangen sei. In $3_{0}$ werde ein Zweig von $G_{n}^{*}(\zeta, z)$ als Kernzweig $\hat{G}_{n}^{*}(\zeta, z)$ festgelegt. Das Mehrdeutigkeitsverhalten von $G_{n}^{*}(\zeta, z)$ in $\overline{3}_{v(n)}$ ist bestimmt durch

$$
\begin{aligned}
& \mathfrak{S}_{\rho}\left\{G_{n}^{*}(\zeta, z)\right\}=g_{n_{\varrho}}^{*}(z)+G_{n}^{*}(\zeta, z), \quad \varrho \leqq k_{v(n)}, \\
& \mathfrak{S}_{\sigma}^{*}\left\{G_{n}^{*}(\zeta, z)\right\}=c_{\sigma} \cdot 2 \pi i+G_{n}^{*}(\zeta, z), \quad c_{\sigma} \operatorname{ganz}, \sigma \leqq l_{\nu(n)} \text {. }
\end{aligned}
$$

1 Für die folgende Überlegung sei angenommen, dass $\Re$ nicht einfach zusammenhängend, also $k_{1} \geqq \mathrm{I}$, ist. Bei einfachem Zusammenhang von $\Re$ vereinfacht sich der folgende Gedankengang. In diesem Falle sind alle $M_{n}^{*}(\zeta, z)$ eindeutig; die Ungleichungen (IO), (I3) fallen fort, es wird lediglich (12) benötigt. 
Hierin ist

$$
g_{n \varrho}^{*}(z)=\log m_{n \varrho}^{*}(z)
$$

(für einen bestimmten Zweig des Logarithmus), insbesondere bleibt $g_{n \varrho}^{*}(z)$ nach Satz 2 in $\overline{\mathfrak{R}}_{v(n)}^{*}$ eindeutig.

Sei ein $\varepsilon_{n}>0$ vorgegeben. Wir können $g_{n \varrho}^{*}(z)$ durch eine auf $\Re^{*}$ eindeutige reguläre Funktion $h_{n_{\ell}}(z)$ approximieren, sodass in $\bar{\Re}_{v(n)}^{*}$ gilt

Ferner setzen wir in $\overline{3}_{v(n)}$ :

$$
\left|g_{n_{0}}^{*}(z)-h_{n \rho}(z)\right|<\frac{\varepsilon_{n}}{\lambda_{n} \cdot k_{v(n)}} .
$$

$$
{ }^{*} G_{n}(\zeta, z)=G_{n}^{*}(\zeta, z)-\sum_{\rho=1}^{k_{v}(n)} I_{\rho}(\zeta) \cdot g_{n_{\rho}}^{*}(z)-\sum_{\sigma=1}^{l_{v}(n)} I_{\sigma}^{*}(z) \cdot c_{\sigma} \cdot 2 \pi i
$$

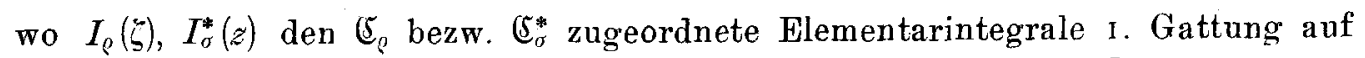
$\Re$ bezw. $\Re^{*}$ bedeuten. ${ }^{*} G_{n}(\zeta, z)$ bleibt bei Fortsetzung innerhalb $\bar{\Omega}_{\nu(n)}$ eindeutig; speziell gelte in $3_{0}$ :

$$
{ }^{*} G_{n}(\zeta, z)=\hat{G}_{n}^{*}(\zeta, z)-\sum_{\rho=1}^{k_{v}(n)} \hat{I}_{0}(\zeta) \cdot g_{n \varrho}^{*}(z)-\sum_{\sigma=1}^{I_{y}(n)} \hat{I}_{\sigma}^{*}(z) \cdot c_{\sigma} \cdot 2 \pi i
$$

dabei bezeichnen $\hat{I}_{0}(\zeta)$ und $\hat{I}_{\sigma}^{*}(z)$ bestimmte, als Kernzweige festgelegte Zweige von $I_{Q}(\zeta)$ bezw. $I_{\sigma}^{*}(z)$. Die $I_{\varphi}(\zeta)$ denken wir uns in folgender Weise normiert: Die Kurve $\mathfrak{S}_{\varrho}$ gehöre zur Homologiebasis von. $\Re_{\tau(\rho)+1}$, nicht aber zu der von $\Re_{\tau(\varrho)}$, es sei also $k_{\tau(\varrho)}<\varrho \leqq k_{\tau(\varrho)+1}$. In $\Re_{\tau(\varrho)}$ bleibt $I_{\ell}(\zeta)$ bei Fortsetzung eindeutig. Wie im Beweise von Satz I kann nun $I_{Q}(\zeta)$ nötigenfalls so abgeändert werden, dass in $\bar{\Re}_{\tau(\rho)}$ gilt (wir bezeichnen die abgeänderte Funktion wieder mit $I_{\rho}(\zeta)$ ):

$$
\left|\hat{I}_{o}(\zeta)\right|<\mathrm{I}
$$

wir denken uns $I_{\rho}(\zeta)$ so gewählt.

Es sei weiter $H_{n}(\zeta, z)$ eine in $3=\Re \times \Re^{*}$ eindeutige reguläre Funktion, für die in $\bar{\bigotimes}_{v\langle n\}}$ gilt

$$
\left|{ }^{*} G_{n}(\zeta, z)-H_{n}(\zeta, z)\right|<\frac{\varepsilon_{n}}{\lambda_{n}}
$$

eine solche Funktion existiert nach dem in Abschnitt I zitierten Approximationssatz. Wir bilden dann

$$
\begin{aligned}
& G_{n}(\zeta, z)={ }^{*} G_{n}(\zeta, z)-H_{n}(\zeta, z)+\sum_{\varrho=1}^{k_{v}(n)} I_{Q}(\zeta) \cdot\left[g_{n_{Q}}^{*}(z)-h_{n_{\ell}}(z)\right] \\
& \equiv G_{n}^{*}(\zeta, z)-H_{n}(\zeta, z)-\sum_{\rho=1}^{k_{\nu}(n)} I_{\varrho}(\zeta) \cdot h_{n_{\ell}}(z)-\sum_{\sigma=1}^{l_{\nu(n)}} I_{\sigma}^{*}(z) \cdot c_{\sigma} \cdot 2 \pi i
\end{aligned}
$$


Der Kernzweig von $G_{n}(\zeta, z)$ sei

$$
\hat{G}_{n}(\zeta, z)=\hat{G}_{n}^{*}(\zeta, z)-H_{n}(\zeta, z)-\sum_{p=1}^{k_{v}(n)} \hat{I}_{\vartheta}(\zeta) \cdot h_{n q}(z)-\sum_{\sigma=1}^{l_{v}(n)} \hat{I}_{\sigma}^{*}(z): c_{\sigma} \cdot 2 \pi i
$$

Setzen wir abkürzend noch

$$
\begin{gathered}
{ }^{\prime} G_{n}(\zeta, z)={ }^{*} G_{n}(\zeta, z)-H_{n}(\zeta, z), \\
g_{n}(\zeta, z)=g_{n^{*}}^{*}(z)-h_{n \varrho}(z),
\end{gathered}
$$

so sind also ' $G_{n}(\zeta, z), g_{n_{\vartheta}}(z)$ in $\overline{\mathscr{B}}_{v(n)}$ bezw. in $\bar{\Re}_{v(n)}^{*}$ regulär und eindeutig, und es gilt

$$
\left.\begin{array}{l}
G_{n}(\zeta, z)={ }^{\prime} G_{n}(\zeta, z)+\sum_{\rho=1}^{k_{\nu}(n)} I_{\varrho}(\zeta) \cdot g_{n \varrho}(z) \\
\left.\right|^{\prime} G_{n}(\zeta, z) \mid<\frac{\varepsilon_{n}}{\hat{\lambda}_{n}}
\end{array}\right\} \text { in } \bar{\beta}_{v(u)}
$$

$$
\left|g_{n_{9}}(z)\right|<\frac{\varepsilon_{n}}{\lambda_{n} \cdot k_{v}(n)} \text { in } \bar{\Re}_{\nu(n)}^{*}
$$

Sei nun

$$
\begin{aligned}
M_{n}(\zeta, z) & =e^{\left(Q_{n}(\xi, z)\right.} \\
& \equiv M_{n}^{*}(\zeta, z) \cdot e^{\left.-H_{n}(\xi, z)-\sum_{\rho=1}^{k_{\nu(n)}} I_{\rho}(\zeta) \cdot h_{n} \rho\right)-\sum_{\sigma=1}^{I_{\nu}(n)} I_{\sigma}^{*}(z) \cdot c_{\sigma} \cdot 2 \pi i}
\end{aligned}
$$

Als Kernzweig von $M_{n}(\zeta, z)$ sei

$$
\hat{M}_{n}(\zeta, z)=e^{\hat{\theta}_{n}(\zeta, z)}
$$

festgesetzt. - Die Zahlen $\varepsilon_{n}>0$ seien so gewählt, dass $\sum_{n} \varepsilon_{n}$ konvergiert. Wir behaupten, dass das Produkt

$$
\prod_{n=1}^{\infty}\left\{M_{n}(\zeta, z)\right\}^{i_{n}}
$$

im oben angegebenen Sinne konvergiert.

Um das zu zeigen, wählen wir irgendein einfach zusammenhängendes Gebiet (SF ganz im Innern von 3 ; dann gilt für ein $\mu>\mathrm{I}: \mathfrak{G} \ll \overline{\bar{B}}_{\mu}$. Wir lassen alle $M_{n}(\zeta, z)$ ausser Betracht, die in $\overline{\boldsymbol{g}}_{\mu}$ verschwinden. Für jeden Faktor des Restproduktes 


$$
\prod_{n=n_{0}}^{\infty}\left\{M_{n}(\zeta, z)\right\}^{i_{n}}
$$

gilt dann jeweils in $\overline{3}_{r}(m)$, nach ( 11$)$ :

$$
\log \left\{M_{n}(\zeta, z)\right\}^{\lambda_{n}}=\lambda_{n} \cdot{ }^{\prime} G_{n}(\zeta, z)+\lambda_{n} \cdot \sum_{\varrho=1}^{k_{v}(n)} I_{\varrho}(\zeta) \cdot g_{n_{\rho}}(z)
$$

(wo links und rechts geeignete Zweige der Funktionen zu wählen sind). Dabei ist jedenfalls $v(n) \geqq \mu$. Speziell ist in $\overline{3} u$ für geeignete Zweige der beteiligten Funktionen

$$
\left.\log \left\{M_{n}(\zeta, z)\right\}\right\}^{\lambda_{n}}=\lambda_{n} \cdot G_{n}(\zeta, z)+\lambda_{n} \cdot \sum_{\varrho=1}^{k_{\mu}} I_{\varrho}(\zeta) \cdot g_{n \varrho}(z)+\lambda_{n} \cdot \sum_{\rho=k_{\mu}+1}^{k_{v}(n)} \hat{I}_{\varrho}(\zeta) \cdot g_{n \varrho}(z)
$$

wobei die letzte Summe rechts fortfällt, falls $k_{\mu}=k_{v(n)}$ ist. $^{1}$ In (S) gilt dann:

$$
\begin{aligned}
& \left|\log \left\{M_{n}(\zeta, z)\right\}^{\lambda_{n}}\right| \leqq\left|\lambda_{n} \cdot{ }^{\prime} G_{n}(\zeta, z)\right|+\sum_{\varrho=1}^{k_{u}}\left|I_{\varrho}(\zeta)\right| \cdot\left|\lambda_{n} g_{n \varrho}(z)\right| \\
& +\sum_{\varrho=k_{\mu}+1}^{k_{v}(n)}\left|\hat{I}_{0}(\zeta)\right| \cdot\left|\lambda_{n} \cdot g_{n g}(z)\right| \\
& \leqq \varepsilon_{n}+\frac{\varepsilon_{n}}{k_{v(n)}} \cdot \sum_{\ell=1}^{k_{\mu}}\left|I_{\ell}(\zeta)\right|+\left(k_{v(n)}-k_{\mu}\right) \cdot \frac{\varepsilon_{n}}{k_{\nu(n)}} \\
& \text { [nach (12), (I 3), (10)] } \\
& \leqq\left[2+\sum_{\rho=1}^{k_{\mu}}\left|I_{Q}(\zeta)\right|\right] \cdot \varepsilon_{n}
\end{aligned}
$$

also:

$$
\sum_{n=n_{1}}^{\infty}\left|\log \left\{M_{n}(\zeta, z)\right\}^{\hat{\lambda}_{n}}\right| \leqq\left[2+\sum_{\ell=1}^{k_{\mu}}\left|I_{0}(\zeta)\right|\right] \cdot \sum_{n=n_{1}}^{\infty} \varepsilon_{n}
$$

für jedes $n_{1} \geqq n_{0}$. Daraus folgt die behauptete absolute und gleichmässige Konvergenz des unendlichen Produktes. Wir setzen

Dann ist

$$
\tilde{M}(\zeta, z)=\prod_{n=1}^{\infty}\left\{M_{n}(\bullet, z)\right\}^{\hat{\lambda}_{n}}
$$

${ }^{1}$ Falls $\Re$ einfach zusammenhängend ist, bleibt nur das erste Glied rechts stehen. 


$$
Q(\zeta, z)=\frac{M(\zeta, z)}{\tilde{M}(\zeta, z)}
$$

eine in 3 reguläre, nichtverschwindende m. a. Funktion. Wir ziehen $Q(\zeta, z)$ in das Produkt hinein, indem wir bilden

dann gilt

$$
M_{0}(\zeta, z)=Q(\zeta, z) \cdot M_{1}(\zeta, z)
$$

$$
M(\zeta, z)=\prod_{n=0}^{\infty}\left\{M_{n}(\zeta, z)\right\}^{x_{n}}
$$

mit $x_{0}=\mathrm{I}, x_{1}=\lambda_{1}-\mathrm{I}, x_{n}=\lambda_{n}$ für $n \geqq 2$.

\section{6) Analytische Flächen zu vorgegebenen charakteristischen Schnittzahlen.}

Es sei jedem Zykel $\mathfrak{I}_{\rho \sigma}=\mathfrak{S}_{\rho} \times \mathfrak{S}_{\sigma}^{*}$ der durch die $\mathfrak{C}_{\rho}$, $\mathfrak{C}_{\sigma}^{*}$ bestimmten zweidimensionalen Homologiebasis in 3 eine ganze Zahl $a_{\rho \sigma}$ beliebig zugeordnet. Dann existiert stets in 3 , wie wir zeigen wollen, eine Menge $\mathfrak{M}$ von analytischen Flächen, die sich im Innern von 3 nicht häufen, mit den $a_{q \sigma}$ als charakteristischen Schnittzahlen.

Hierzu benötigen wir folgenden

Hilfssatz: $E s$ sei $k_{v}<\varrho_{1} \leqq k_{v+1}, l_{\mu}<\sigma_{1} \leqq l_{\mu+1}$. Dann gitt es in 3 eine dort singularitätenfreie Menge $\mathfrak{F}_{\rho_{1} \sigma_{1}}$ ergänzter analytischer Flächenstücke ${ }^{1}$ mit folgenden Eigenschaften:

I) Werden die irreduziblen Bestandteile von $\widetilde{J}_{\ell_{1} \sigma_{1}}$ mit der Ordnung I versehen und natürlich orientiert, so gilt für die charakteristischen Schnittzahlen $b_{\rho \sigma}$ von $\mathfrak{F}_{\hat{0}_{1} \sigma_{1}}$ :

$$
\begin{aligned}
& b_{\rho o}=0 \text { für }\left\{\begin{array}{l}
\varrho \neq \varrho_{1}, \sigma \text { beliebig; } \\
\varrho=\varrho_{1}, \sigma<\sigma_{1} ;
\end{array}\right. \\
& b_{\rho \sigma}=a_{\rho \sigma} \text { für } \varrho=\varrho_{1}, \sigma \geqq \sigma_{1} .
\end{aligned}
$$

2) $\mathfrak{F}_{\rho_{1} \sigma_{1}}$ dringt nicht in $\mathfrak{R}_{v} \times \Re_{\mu}^{*}$ ein.

1 Zum Begriff des ergänzten analytischen Flächenstückes siehe H. BEHNkE und P. ThULLEN, Theorie der Funktionen mehrerer komplexer Veränderlichen, Ergebnisse der Mathematik und ihrer Grenzgebiete, Bd. 3, Berlin 1934. 
Beweis: Wir wählen in $\Re$ zu $\mathfrak{S}_{\rho_{1}}$ ein Elementarintegral I. Gattung $I_{\rho_{1}}(\zeta)$, ferner in $\Re^{*}$ ein Integral I. Gattung $A_{Q_{1} \gamma_{1}}(z)$ mit den wie oben festgelegten $b_{\rho_{1} \sigma}$ als Periodizitätsmoduln. Wegen $\varrho_{1}>k_{v}, \sigma_{1}>l_{\mu}$ ist jeder Zweig von $I_{\rho_{1}}(\zeta)$ in $\Re_{v}$ bezw. jeder $Z_{w e i g}$ von $A_{Q_{1} \sigma_{l}}(z)$ in $\Re_{u}^{*}$ eindeutig. Wir legen je einen $Z$ weig von $I_{\rho_{1}}(\zeta)$ in $\Re_{v}$ bezw. von $A_{\rho_{1} \sigma_{1}}(z)$ in $\Re_{l}^{*}$ als Kernzweige $\hat{I}_{\rho_{1}}(\zeta)$ bezw. $\hat{A}_{\rho_{1} \sigma_{1}}(z)$ fest. Sei ein $\varepsilon>0$ vorgegeben. Dann können, ähnlich wie früher, $I_{\rho_{1}}(\zeta)$ und $A_{Q_{1} \sigma_{1}}(z)$ so normiert werden, dass gilt

$$
\begin{aligned}
& \left|\hat{I}_{Q_{1}}(\zeta)\right|<\varepsilon \text { in } \Re_{v}, \\
& \left|\hat{A}_{Q_{1} \sigma_{1}}(z)-\frac{1}{2}\right|<\varepsilon \text { in } \Re_{\mu}^{*} .
\end{aligned}
$$

$\mathrm{Zu}$ beliebig herausgegriffenen Zweigen von $I_{0_{1}}(\zeta)$ und $A_{Q_{1} \sigma_{1}}(z)$ gibt es dann, da alle Periodizitätsmoduln ganzzahlig sind, geeignete ganze Zahlen $p, q$, sodass gilt

$$
\left|I_{p_{1}}(\zeta)-p\right|<\varepsilon \text { in } \Re_{v}
$$

Wir wählen etwa $\varepsilon<\frac{1}{4}$.

Nunmehr ziehen wir die im Abschnitt 3 (Beweis zu Satz 4) eingeführte Funktion $H(\tilde{\zeta}, \tilde{z})$ heran, die im schlichten Zylindergebiet $\tilde{\mathfrak{Z}}:\{0<|\tilde{\zeta}|<\infty ; 0<|\tilde{z}|<\infty\}$ als reguläre m. a. Funktion mit der Nullstellenflïche $\tilde{z}=\tilde{\zeta}^{i}$ definiert ist. Wir bilden in $8=\Re \times \Re^{*}$ :

$$
G_{\hat{O}_{1} \sigma_{1}}(\zeta, z)=H\left(e^{2 \pi i I_{0_{1}}(\xi)}, e^{2 \pi i A_{\hat{j}_{1} \sigma_{1}}(z)}\right) .
$$

$G_{\ell_{1} \sigma_{1}}(\zeta, z)$ ist in 3 eine reguläre m. a. Funktion, und zwar gilt:

$$
\begin{aligned}
& \mathfrak{S}_{2}\left\{G_{Q_{1} \sigma_{1}}(\zeta, z)\right\}=G_{Q_{1} \sigma_{1}}(\zeta, z) \text { für } \varrho \neq \varrho_{1}, \\
& \mathfrak{S}_{Q_{1}}\left\{G_{Q_{1} \sigma_{1}}(\zeta, z)\right\}=e^{2 \pi i}{ }_{\Delta \varrho_{1} \sigma_{1}}(z) \cdot G_{Q_{1} \sigma_{1}}(\zeta, z) .
\end{aligned}
$$

Die Nullstellengesamtheit von $G_{\rho_{1} \sigma_{1}}(\zeta, z)$ bildet nun eine Menge von analytischen Flächen mit den angegebenen Eigenschaften, sie sei mit $\mathfrak{F}_{0_{1} \sigma_{1}}$ bezeichnet.

Zunächst stimmen die charakteristischen Schnittzahlen von $\mathfrak{F}_{O_{1} \sigma_{1}}$ nach Satz 4 mit den vorgegebenen $b_{\xi \sigma}$ überein. Ferner gilt für die Punkte $\mathfrak{B}(\zeta) \times \mathfrak{B}(z)$ auf $\widetilde{\mho}_{Q_{1} \sigma_{1}}$ :

also

$$
e^{2 \pi i \cdot I_{\rho_{1}}(\because)}=e^{-i\left[2 \pi i \cdot A_{\rho_{1} \sigma_{1}}(:)\right]},
$$

$$
I_{0_{1}}(\zeta)+k=-i \cdot A_{Q_{1} \sigma_{1}}(z) ; k \text { eine ganze Zahl. }
$$

Diese Beziehung kann jedoch, wegen (14) und (I 5 ) mit $\varepsilon<\frac{1}{4}$, für kein $\mathfrak{P}(\zeta) \times$ 
$\times \mathfrak{P}(z)$ aus $\mathfrak{R}_{v} \times \mathfrak{R}_{\mu}^{*}$ erfüllt sein. Daher dringt $\mathfrak{F}_{\mathfrak{o}_{1} \sigma_{1}}$ nicht in $\mathfrak{R}_{v} \times \mathfrak{R}_{\mu}^{*}$ ein, womit der Hilfssatz bewiesen ist.

Nunmehr können wir eine gesuchte Menge $\mathfrak{M}$ von analytischen Flächeri mit deu charakteristischen schnittzahlen $a_{\rho \sigma}$ in 8 konstruieren. Besteht die eindimensionale Homologiegruppe von $\Re$, bezw. die von $\Re^{*}$, nur aus dem Nullelement, sind also alle $a_{\mathfrak{l} \sigma}=0$, so ist schon die Fläche $\mathfrak{P}(\zeta)=\mathfrak{P}\left(\zeta_{0}\right), \mathfrak{P}\left(\zeta_{0}\right)$ ein fester Punkt aus $\mathfrak{R}$, eine gesuchte Menge $\mathfrak{M}$. Anderenfalls verfahren wir wie folgt: Wir bilden die Flächenstiickmengen $\mathfrak{F}_{x x}, x=\mathrm{I}, 2, \ldots$, mit den im Hilfssatz angegebenen Eigenschaften. ${ }^{1}$ Die charakteristischen Schnittzahlen eines $\widetilde{F}_{x x}$ stimmen mit den Elementen der $x$. Zeile der Matrix $\left(a_{\varrho}\right)$ überein, wobei jedoch die ersten $(x-1)$ Elemente dieser Zeile durch Nullen ersetzt sind. Die Vereinigungsmenge

$$
\tilde{\mathfrak{M}}=\sum_{x} \widetilde{\mho}_{x x}
$$

besitzt dann als charakteristische Schnittzahlen die Elemente oberhalb und einschliesslich der Hauptdiagonalen der Matrix $\left(a_{0}\right)$ und sonst Nullen, d. h.: Die zu den $\mathfrak{I}_{\rho}=\mathfrak{\complement}_{\rho} \times \mathfrak{S}_{\sigma}^{*}$ mit $\varrho \leqq \sigma$ gehörigen charakteristischen Schnittzahlen stimmen mit den $a_{0 \sigma}$ überein, während die übrigen Schnittzahlen sämtlich Null sind. Ferner häufen sich die $\mathfrak{F}_{x_{x}}$ innerhalb 3 nicht, da mit einem $3_{v}=\Re_{v} \times \Re_{v}^{*}$ höchstens diejenigen endlich vielen $\mathfrak{F}_{x x}$ Punkte gemeinsam haben, für welche $x \leqq k_{v}$ oder $x \leqq l_{v}$ ist. Entsprechend gibt es auf Grund des Hilfssatzes (bei seiner An. wendung sind jetzt die Variablen zu vertauschen) in 3 Mengen $\mathfrak{F}_{2.2}^{*}$ von analytischen Flächenstücken, die sich innerhalb von 3 nicht häufen und deren Ver. einigungsmenge

$$
\mathfrak{M}^{*}=\sum_{i} \mathfrak{F}_{i \lambda}^{*}
$$

als charakteristische Schnittzahlen die Elemente unterhalb der Hauptdiagonalen der Matrix $\left(a_{\rho \sigma}\right)$ und sonst Nullen hat. Also ist die Menge

$$
\mathfrak{M}=\tilde{\mathfrak{M}}+\mathfrak{M}^{*}
$$

eine gesuchte Menge $\mathfrak{M}$.

Daher ist bewiesen

Satz 7: In Zylindergebiet $B=\mathfrak{R} \times \Re^{*}$ existiert zu beliebig vorgegebenen charakteristischen Schnittzahlen stets eine Menge $\mathfrak{M}$ von ergänzten analytischen Flächenstïcken, die sich im Innern von 3 nicht häufen.

${ }^{1}$ Ist $x>k_{v}$ für alle $v$ oder $x>l_{\mu}$ für alle $\mu$, so sei $\tilde{\partial}_{x x}$ die leere Menge.

14-642136 Acta mathematica. 83 
Es ist klar, dass $\mathfrak{M}$ dann auch durch eine Consinsche Verteilung 2. Art von in 3 regulären Ortsfunktionen gegeben werden kann.

Fast unmittelbar folgt:

Satz 8: Im Zylindergebiet $3=\mathfrak{\Re} \times \mathfrak{R}^{*}$ sei cine Cousinsche Verteilung 2. Art $V$ von regulären Ortsfunktionen vorgegeben. Dann existiert stets eine in 8 ein. deutige reguläre Funktion, die auf den durch $V$ vorgeschriebenen Nullstellenfächen mindestens in der vorgeschriebenen Ordnung verschwindet.

Beweis: Die durch $V$ vorgeschriebene, mit Ordnungen und natürlicher Orientierung versehene Menge von Nullstellenflächen sei $\mathfrak{R}$. Wir wählen eine mit Ordnungen und natürlicher Orientierung versehene Menge $\tilde{\mathfrak{R}}$ analytischer Flächen, die sich im Innern von 8 nicht häufen, sodass die charakteristischen Schnittzahlen der Vereinigungsmenge

$$
\mathfrak{M}=\mathfrak{R}+\tilde{\mathfrak{M}}
$$

sämtlich Null sind: das ist nach Satz 7 möglich. Zu M gehört nach der Folgerung aus Satz 5 eine in $B$ eindeutige reguläre Funktion, die genau auf $\mathfrak{M}$ in den vorgeschriebenen Ordnungen verschwindet, und diese Funktion hat die geforderte Eigenschaft.

Verlangt man also von einer Lösungsfunktion $F(\zeta, z)$ zu einer vorgegebenen Cousinschen Verteilung 2. Art in $8=\mathfrak{R} \times \mathfrak{R}^{*}$ nur, dass jeweils in $\mathfrak{U}(\boldsymbol{P})$ gelten soll

$$
F(\zeta, z)=L_{P}(\zeta, z) \cdot f_{P}(\zeta, z)
$$

wo $L_{P}(\zeta, z)$ in $\mathfrak{U}(P)$ regulär ist, so existiert stets eine in 3 eindeutige reguläre Lösung $F(\zeta, z)$. In diesem erweiterten Sinne ist jedes Cousinsche Problem 2. Art in einem Zylindergebiet durch eindeutige Funktionen lösbar. Das hat folgende bemerkenswerte Konsequenz:

Satz 9: Jede im Zylindergebiet $3=\Re \times \Re^{*}$ eindeutige meromorphe Funktion $G(\zeta, z)$ gestattet in 3 eine Quotientendarstellung

$$
G(\zeta, z)=\frac{F_{1}(\zeta, z)}{F_{\mathrm{a}}(\zeta, z)}
$$

wo $F_{1}(\zeta, z)$ und $F_{2}(\zeta, z)$ in 8 eindeutige reguläre Funktionen sind.

Beweis: Sei $\mathfrak{R}$ die mit Ordnungen und Orientierung versehene Polfächenmenge von $G(\zeta, z)$. Es gibt nach Satz 8 eine in 8 reguläre eindeutige Funktion 
$F_{2}(\zeta, z)$, die auf $\mathfrak{R}$ mindestens in den dort durch $G(\zeta, z)$ vorgegebenen Polordnungen verschwindet. Die Funktion

$$
F_{1}(\zeta, z)=G(\zeta, z) \cdot F_{9}(\zeta, z)
$$

ist daher in alle Punkte von 8 regulïr fortsetzbar und bleibt dort eindeutig; also gilt in 8

w. z. b. w.

$$
G(\zeta, z)=\frac{F_{1}(\zeta, z)}{F_{2}(\zeta, z)}
$$

\section{7) Multiplikative automorphe Funktionen in $(2 n)$-dimensionalen Zylindergebieten.}

$\mathrm{Zu}$ Grunde gelegt werde jetzt ein nichtgeschlossenes Zylindergebiet $3=$ $=\mathfrak{R}^{(1)} \times \cdots \times \mathfrak{R}^{(n)}$, d. h. ein direktes Produkt nichtgeschlossener Riemannscher Flächen $\mathfrak{R}^{(2)}, \ldots, \mathfrak{R}^{(n)}$ über den $z_{1}-, \ldots, z_{n}$-Ebenen. In jedem $\mathfrak{R}^{(j)}$ sei eine eindimensionale Homologiebasis, bestehend aus orientierten geschlossenen Kurven $\mathfrak{S}_{o}^{(j)}$ mit den üblichen Eigenschaften, gewählt. Die Zykel $\mathfrak{\Im}_{\rho}^{(i)} \times \mathfrak{\Im}_{\sigma}^{(j)}, i<j$, multipliziert mit $n-2$ festen Punkten aus den $\mathfrak{R}^{(k)}, k \neq i, j$, bilden dann eine zweidimensionale Homologiebasis $\left\{\mathfrak{I}_{\rho \sigma}^{(i, j)}\right\}$ in 8 .

Es ist zweckmässig, eine Funktion $M\left(z_{1}, \ldots, z_{n}\right)$ eine in $3=\Re^{(1)} \times \cdots \times \Re^{(n)}$ reguläre multiplikative a tom orphe Funktion zu nennen, wenn $M\left(z_{1}, \ldots, z_{n}\right)$ folgende Eigenschaften besitzt:

1) $M\left(z_{1}, \ldots, z_{n}\right)$ ist in 8 uneingeschränkt regulär fortsetzbar.

2) Bei festen $\mathfrak{P}\left(z_{1}\right), \ldots, \mathfrak{P}\left(z_{n-1}\right)$ und variablem $\mathfrak{P}\left(z_{n}\right)$ bleibt $\boldsymbol{M}\left(z_{1}, \ldots, z_{n}\right)$ eindeutig.

3) Durchläuft $\mathfrak{B}\left(z_{i}\right), i \neq n$, ein $\mathfrak{S}_{p}^{(i)}$ in $\mathfrak{R}^{(i)}$ und werden die übrigen Variablen $\mathfrak{P}\left(z_{j}\right)$ festgehalten, so erhält $M\left(z_{1}, \ldots, z_{n}\right)$ einen Faktor $\prod_{j>i} m_{\rho}^{(i, j)}\left(z_{j}\right)$, symbolisch :

$$
\mathfrak{C}_{p}^{(i)}\left\{\boldsymbol{M}\left(z_{1}, \ldots, z_{n}\right)\right\}=\prod_{j>i} m_{\rho}^{(i, j)}\left(z_{j}\right) \cdot M\left(z_{1}, \ldots, z_{n}\right) ;
$$

dabei ist $m_{Q}^{(i, j)}\left(z_{j}\right)$ in $\Re^{(j)}$ regulär, eindeutig und ungleich Null und hängt nur von der Homologieklasse von $\mathbb{S}_{\rho}^{(i)}$ in $\mathfrak{R}^{(i)}$ ab.

Wird der Begriff der m. a. Funktion in dieser Weise verallgemeinert, so lassen sich die Ergebnisse der vorangehenden Abschnitte ausnahmslos übertragen. Die Beweise verlaufen entsprechend; wir verzichten auf ihre Durchführung und begnügen uns mit der Angabe der Resultate. 
Jede in 8 vorgegebene Cousinsche Verteilung 2. Art $V$ von regulären Ortsfunktionen besitzt eine reguläre m. a. Funktion $M\left(z_{1}, \ldots, z_{n}\right)$ als Lösungsfunktion, die ohne ,überflüssige Mehrdeutigkeiten“ gewählt werden kann. Unter den charakteristischen Schnittzahlen $a_{\varrho \sigma}^{(i, j)}, i<j$, der durch $V$ bestimmten Menge $\mathfrak{M}$ von orientierten und mit Ordnungen versehenen Nullstellenflächen verstehen wir die Schnittzahlen $S\left(\mathfrak{M}, \mathfrak{I}_{\varrho}^{(i, j)}\right.$. Dann gilt für die Multiplikatoren $m_{\varrho}^{(i, j)}\left(z_{j}\right)$ von $M\left(z_{1}, \ldots, z_{n}\right)$ :

$$
a_{\varrho}^{(i, j)}=\int_{\mathbb{E}_{0}^{(j)}} d \log m_{\varrho}^{(i, j)}\left(z_{j}\right)
$$

Jede in 3 reguläre m. a. Funktion (die insbesondere eindeutig sein kann) lässt sich ferner als (im wesentlichen eindeutiges) Produkt von Primfunktionen darstellen. Dabei wird unter einer Primfunktion eine reguläre m. a. Funktion mit genau einer irréduziblen Nullstellenfläche I. Ordnung verstanden. - Zu vorge-

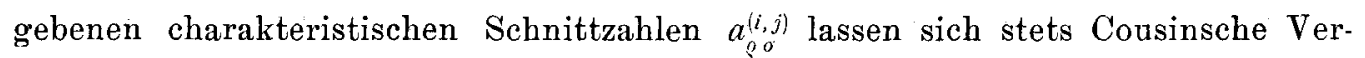
teilungen 2. Art von regulären Ortsfunktionen angeben. Daraus folgt, dass eine in 3 meromorphe eindentige Funktion dort eine Darstellung als Quotient von in 3 eindeutigen regulïren, jedoch nicht notwendig teilerfremden Funktionen gestattet. 